\title{
Fasten: Zur Popularität einer (religiösen) Praktik
}

\author{
Patrick Heiser (iD
}

Eingegangen: 15. Dezember 2020 / Überarbeitet: 30. August 2021 / Angenommen: 9. September 2021 / Online publiziert: 29. September 2021

(C) Der/die Autor(en) 2021

Zusammenfassung Fasten weist nicht nur eine jahrtausendealte Tradition auf, sondern ist bis heute Bestandteil aller Weltreligionen. Auch in spätmodernen Gesellschaften wird es von vielen Menschen regelmäßig praktiziert; eine Beschränkung auf bestimmte Altersgruppen oder Schichten lässt sich nicht erkennen. Dies zeigt die Analyse einer standardisierten Online-Befragung von 1908 Personen aus Deutschland, deren Ergebnisse im vorliegenden Aufsatz vorgestellt und diskutiert werden. Ausgeleuchtet wird dabei eine hohe Gestaltungs- und Deutungshoheit des fastenden Individuums. Insbesondere die nachwachsende Generation von Fastenden bringt neue Verzichtsoptionen und Fastenmotivationen in die tradierte Fastenpraxis ein. Eine deskriptive Analyse arbeitet drei Konstanten des zeitgenössischen Fastens heraus: einen temporären Verzicht auf Genussmittel wie Alkohol und Süßigkeiten, eine subjektbezogene Fastenmotivation und eine fastenbedingte Steigerung des körperlichen Wohlbefindens. Anhand einer Regressionsanalyse werden drei zentrale Determinanten identifiziert, von denen sowohl die Gestaltung als auch die Effekte des Fastens abhängen: das Alter, die Konfessionszugehörigkeit und die Religiosität. Anhand dieser Befunde wird die Frage diskutiert, ob und inwiefern Fasten auch unter den säkularen Bedingungen der späten Moderne als religiöse Praktik konzeptualisiert werden kann. In Anschluss an den Lived-religion-Ansatz wird es als gelebte religiöse Praktik verstanden, deren Popularität sich durch ein spezifisches Zusammenspiel von individuellen Gestaltungsspielräumen und institutioneller Evidenzsicherung erklären lässt. Nicht irgendwann nämlich fastet die überwiegende Mehrheit der Befragten, sondern in konfessionell proklamierten Fastenzeiten mit langer Tradition.

Schlüsselwörter Fasten · Religiöse Praktiken · Gelebte Religion

Patrick Heiser $(\bowtie)$

Institut für Soziologie, Lehrgebiet Soziologie II: Soziologische Gegenwartsdiagnosen,

FernUniversität in Hagen, 58094 Hagen, Deutschland

E-Mail: patrick.heiser@fernuni-hagen.de 


\section{Fasting: Popularity of a (religious) practice}

Abstract Fasting not only has a tradition dating back thousands of years, but is still part of all world religions. Even in late modern societies it is practiced regularly by many people; a limitation to certain age groups or social classes cannot be observed. This shows an online survey of 1,908 Germans, the results of which are presented and discussed in this paper. It shows a remarkable individual autonomy of shaping and interpreting practices of fasting. In particular, an upcoming generation of fasting people is introducing new renunciation options and motivations into the tradition of fasting. A descriptive analysis identifies three constants of contemporary fasting: a temporary renunciation of stimulants such as alcohol and sweets, a subjectrelated fasting motivation, and a fasting-related increase in physical well-being. Applying a regression analysis, three central determinants can be identified on which both the shaping and the effects of fasting depend: age, denominational affiliation, and religiosity. Based on these findings, the question is discussed whether and to what extent fasting can be conceptualized as a religious practice even under the secular conditions of late modernity. Following the lived-religion approach, fasting is understood as a religious practice whose popularity can be explained by a specific interplay of individual autonomy and institutional ensuring of evidence. Namely, most of the participants do not fast at any time whatsoever, but in institutionally proclaimed periods with long tradition.

Keywords Fasting $\cdot$ Religious practices $\cdot$ Lived religion

\section{Fasten und Religion}

„Fasten liegt im Trend“ titeln verschiedene Zeitungen der Funcke Mediengruppe zu Beginn der vorösterlichen Fastenzeit des Jahres 2019 (NRZ 2019). Diese journalistische Einschätzung basiert auf einer Online-Befragung des Meinungsforschungsinstituts Civey, der zufolge 36,4\% der Befragten bis Ostern Verzicht üben wollen. Ein freiwilliger und temporärer Verzicht auf (bestimmte) Nahrungsmittel, Genussmittel und/oder Konsumgüter, der während der gesamten Fastenzeit oder nur zu bestimmten Tageszeiten erfolgen kann, scheint heutzutage also durchaus beachtliche Popularitätswerte aufzuweisen. Historisch belegt ist er bereits durch antike Quellen (Arbesmann 1929; Musurillo 1956); Fasten lässt sich, so die Feststellung Tamneys (1980, S. 129), gar in der gesamten bisherigen Menschheitsgeschichte nachweisen. Dies mag auch darin begründet liegen, dass frühe Formen des Fastens auf eine jahreszeitlich bedingte Einschränkung des Nahrungsangebots zurückzuführen waren, die gleichsam für ,natürliche Fastenperioden“ sorgten (Fritzsche 2008, S. 16). Da dieser Umstand im Laufe der menschlichen Evolution zunehmend überwunden werden konnte, änderte sich auch die gesellschaftliche Bedeutung des Fastens. Im Mittelalter war es in erster Linie Symbol für Tod und Wiedergeburt; es diente der Reinigung und Vorbereitung auf den Empfang transzendenter Kräfte. Daher wurde beispielsweise unmittelbar vor einer Priesterweihe oder Krönung gefastet (Hocart 1931, S. 145). In der frühen Moderne verbreitete sich das Fasten über diese spe- 
zifischen Personengruppen des Klerus und Adels hinaus. Dabei wandelte es sich mehr und mehr zum Ausdruck von Frömmigkeit, Buße und Kontrolle über den eigenen Körper; es erhielt seinen bis heute fortbestehenden asketischen Charakter. Die Proklamation von Fastenzeiten sowie die Regulation und Sanktionierung von Fastenpraktiken fielen nun in den Zuständigkeitsbereich religiöser Institutionen. Im Christentum stand dabei die Buße im Vordergrund, im Islam die Frömmigkeit (Wagtendonk 1968).

Das Fasten in je spezifischen Fastenzeiten ist bis heute Bestandteil aller Weltreligionen (Grimm 1996). So zeichnet sich das Judentum ohnehin durch vergleichsweise strenge Ernährungsvorschriften aus, die im Wesentlichen auf die mosaischen Speisegesetze zurückgehen (Suchy 1995). Im jüdischen Glauben sind fünf Fastentage pro Jahr von Bedeutung, darunter insbesondere das Versöhnungsfest Jom Kippur. Unter Bezugnahme auf das Alte Testament erinnert es daran, dass Moses vor seiner göttlichen Offenbarung 40 Tage und Nächte lang am Berg Sinai fastete (2 Mose 34, 38; 3 Mose 16, 29-31). Hinzu kommen die Fastentage zu Assara Betewet, Schiwa'Assar beTammus, Tischa beAw und Zom Gedalja. Vor Jom Kippur und Tischa beAw verzichten Gläubige jeweils knapp 25 Stunden lang völlig auf Essen, Trinken, Rauchen, Arbeit und Geschlechtsverkehr; vor Tischa beAw wird gelegentlich auch für drei Wochen auf Fleisch und Wein verzichtet. An den übrigen jüdischen Fastentagen wird lediglich von Sonnenauf- bis Sonnenuntergang gefastet. Darüber hinaus werden bis heute einige Fastentage für spezifische Personengruppen praktiziert: Beispielsweise fasten Erstgeborene häufig am Tag vor Pessach, um an die Errettung der israelitischen Erstgeborenen zu erinnern, sowie Brautpaare am Tag vor ihrer Hochzeit. ${ }^{1}$

In Bezug auf das Christentum gilt es zu differenzieren: Im Katholizismus und der orthodoxen Kirche hat Fasten bis heute den Charakter einer religiösen Pflicht - wenn auch in mittlerweile abgeschwächter Form. Der Protestantismus hingegen missbilligte das Fasten im Zuge der Reformation (Zwingli 1900), erlaubt es seinen Gläubigen heute jedoch als „hilfreiche Unterstützung des Gebets“ (Setzwein 1997, S. 179). Die bedeutendste Fastenzeit des Christentums stellt die vorösterliche Bußzeit dar ${ }^{2}$, in der Gläubige nach dem Vorbild Jesu 40 Tage lang fasten (Mt 5, 1-14; Mt 6, 17-18). Die exakte Bestimmung der vorösterlichen Bußzeit bedarf der Berücksichtigung einiger theologischer Prämissen: So beginnt die Fastenzeit unmittelbar in Anschluss an die Fastnacht am Aschermittwoch und währt letztlich bis zum Karsamstag. Der Karsamstag selbst zählt aber ebenso wenig zur Fastenzeit wie der Karfreitag und der Aschermittwoch - gleichwohl es sich in beiden Fällen um Fasten- bzw. Abstinenztage handelt. Sonntage hingegen sind keine Fastentage. Daher umfasst die vorösterliche Bußzeit zwar ,nur' 40 Tage, währt aber

\footnotetext{
${ }^{1}$ Seltener findet sich im zeitgenössischen Judentum noch das Wochenfasten an Montagen und Donnerstagen. Traditionell wurde an diesen Tagen die Thora studiert. Um die Konzentration auf die Heilige Schrift zu steigern, sollte jeweils vor der Mittagszeit auf Speisen und Getränke verzichtet werden.

2 Traditionell zählte auch die Adventszeit als christliche Fastenzeit. Darüber hinaus wurde aus dem Judentum das Wochenfasten übernommen, allerdings nicht an Montagen und Donnerstagen, sondern an Mittwochen (dem Tag der Festnahme Jesu) und Freitagen (dem Tag seines Todes). Seltener fasten Katholikinnen und Katholiken heute noch vor besonderen Anlässen, wie den Namenstagen wichtiger Heiliger, oder vor dem Empfang der Eucharistie.
} 
dennoch sechseinhalb Wochen. Traditionell wird in dieser Zeit zwischen Sonnenauf- und Sonnenuntergang völlig auf Speisen und Getränke verzichtet; nach einer Abendandacht soll die einzige Mahlzeit des Tages dann idealerweise aus Wasser und Brot bestehen (Setzwein 1997, S. 178 f.). Traditionelles christliches Fasten ist daher mitunter strenger reguliert als das islamische. In früheren Zeiten wurden Verstöße gegen die Fastengebote streng sanktioniert, beispielsweise durch einen Ausschluss von Osterfest und Abendmahl sowie teils auch durch körperliche Strafen (Teuteberg 1988, S. 370 f.). Da die christliche Fastenpraxis im Neuen Testament jedoch nicht explizit kanonisiert ist, kam es auf Seiten der katholischen Kirche im Zuge des Zweiten Vatikanischen Konzils (1962-1965) sowohl quantitativ (in Hinblick auf die Anzahl verbindlicher Fastentage) als auch qualitativ (in Hinblick auf verbindliche Fastenregeln) zu einer spürbaren Lockerung. Seither gelten lediglich noch der Aschermittwoch und der Karfreitag als verbindliche Fasten- und Abstinenztage im traditionellen Sinne. Die Regeln für die übrige Fastenzeit hingegen verlagerten sich von einem umfangreichen Verzicht auf Nahrungsmittel und Getränke hin zu einem Verzicht auf bestimmte Genussmittel und Konsumgüter. Heute empfiehlt die katholische Kirche (2015), während der Fastenzeit nur eine Mahlzeit pro Tag zu sich zu nehmen, ergänzt um zwei ,kleine Stärkungen“. Die evangelische Kirche (2020) hingegen legt ihren Mitgliedern im Zuge ihrer Fastenkampagne „Sieben Wochen ohne“ nahe, während der vorösterlichen Bußzeit bestimmte Praktiken und Weltsichten abzulegen, um alltägliche Routinen reflektieren zu können. In den vergangenen Jahren hat sie beispielsweise den temporären Verzicht auf Blockaden (2021), Pessimismus (2020) und Lügen (2019) empfohlen.

Im Islam hat das Fasten einen bis heute hohen Stellenwert als weitverbreitete religiöse Praxis. Da es sich neben dem Glaubensbekenntnis, dem Gebet, der Almosengabe und der Pilgerfahrt nach Mekka beim Fasten um eine der fünf Säulen des Islam handelt, ist jede/r erwachsene Muslim/in, der/die dazu körperlich in der Lage ist, von seiner/ihrer Religion angehalten, jährlich während des neunten Monats des islamischen Mondkalenders, dem Ramadan, zu fasten. Unter den fünf Säulen nimmt das Fasten eine herausgehobene Stellung ein: Das Glaubensbekenntnis stellt aufgrund seines grundlegenden Charakters strenggenommen keine Praktik dar; bei Gebet und Almosengabe handelt es sich um alltägliche Praktiken, bei der Pilgerfahrt hingegen um ein lebensgeschichtlich singuläres Ereignis. Das Fasten aber ist weder alltäglich noch singulär, sondern vielmehr der wichtigste ,kollektiv kalendarische Ritus“ (Buitelaar 1993) des islamischen Jahres. Laut der Überlieferung betete der Prophet Mohammed jährlich einen Monat lang in der Abgeschiedenheit des Berges Hara, wo ihm durch den Erzengel Gabriel der Koran offenbart wurde (Sure 2, 183-187). In Erinnerung daran wurde der Ramadan als Fastenmonat festgelegt, in dem Muslim/innen zwischen Sonnenauf- und Sonnenuntergang völlig auf Speisen, Getränke, Rauchen und Geschlechtsverkehr verzichten. Nach Sonnenuntergang wird das Fasten dann gemeinschaftlich und feierlich gebrochen.

Auch in den fernöstlichen Religionen ist das Fasten als religiöse Praxis bekannt. So ist im Hinduismus beispielsweise das Fasten an den sogenannten Ekadashi-Tagen, den jeweils elften Tagen nach jeder Mondhälfte, weit verbreitet. Hinzu kommen das extreme Fasten der Sadhus und die maßvolle Ernährung der Yogi, die als Vorbild dafür gelten, dass Fasten heute zu den zehn Grundsätzen des Yoga zählt. Auch im 
Buddhismus existieren zwar keine mit den monotheistischen Religionen vergleichbaren Fastenregeln. Jedoch wird in vielen thailändischen Klöstern zwischen 12:00 Uhr mittags und dem folgenden Morgen nicht gegessen - und erwachsene Männer buddhistischen Glaubens sind angehalten, zumindest einmal in ihrem Leben wie ein Mönch zu leben. Als Vorbild gilt dabei Buddha, der vor seiner Erleuchtung eine Phase extremer Askese durchlebt hatte, zu der auch das Fasten gehörte.

Zusammenfassend lässt sich festhalten, dass wir es sowohl bei frühmodernen Formen des Fastens als auch beim Fasten in konfessionellen Kontexten mit Praktiken zu tun haben, die vergleichsweise eindeutig als religiös zu klassifizieren sind. Fraglich aber ist, ob das Fasten auch unter den säkularen Bedingungen der späten Moderne und auch beim Vollzug jenseits konfessioneller Kontexte als religiöse Praktik verstanden werden kann. Da (religiöse) Praktiken vom spätmodernen Individuum weitgehend selbstbestimmt gestaltet werden (Luckmann 1991; Gebhardt et al. 2005; Beck 2008), ist zu vermuten, dass zeitgenössisches Fasten in nicht wenigen Fällen mit anderen, eben nicht genuin religiösen Sinnkontexten verknüpft ist. Gleichwohl scheint wenig wahrscheinlich, dass die religiösen Ursprünge und die konfessionellen Traditionen des Fastens ihren Einfluss auf spätmoderne Fastenpraktiken völlig verloren haben. Die vorliegende Studie fragt daher nach dem Zusammenhang von Fasten und Religion. Einerseits verfolgt sie ein deskriptives Erkenntnisinteresse: Angesichts eines rudimentären Forschungsstands soll zunächst beschrieben werden, wer in Deutschland heutzutage wie, warum und mit welchen Effekten fastet. Auf einer analytischen Ebene sollen andererseits die Determinanten zeitgenössischer Fastenpraktiken herausgearbeitet werden, um aus einer religionssoziologischen Perspektive diskutieren zu können, ob und inwiefern spätmodernes Fasten als religiöse Praktik konzeptualisiert werden kann.

\section{Forschungsstand und Hypothesen}

Trotz seiner Tradition und seiner gegenwärtigen Popularität ist das Fasten sowohl im deutschsprachigen Raum als auch international bislang kaum Gegenstand sozialwissenschaftlicher Forschung. Zwar sind die Regale einschlägiger Bibliotheken durchaus gut gefüllt mit Ratgebern zum sogenannten Heil- und Intervallfasten. Derartige Publikationen jedoch sind überwiegend der Kategorie nichtwissenschaftlicher Sachbücher, häufig mit esoterischem Charakter, zuzuordnen; nur gelegentlich zeichnen sie sich durch eine ernährungswissenschaftliche Fundierung aus. Darüber hinaus findet sich eine ganze Reihe kulturhistorischer Publikationen, die sich mit der Entwicklung von Fastenpraktiken befassen (Heun 1972; Wagner 1986; Teuteberg 1988; Harris 1990; Laurioux 1992; Suchy 1995; Grimm 1996), sowie medizinische Literatur zu den physiologischen Folgewirkungen des Fastens (Fritzsche 2008; Ahmad et al. 2012). Systematischer wurde das islamische Fasten in den sozialwissenschaftlichen Blick genommen (Tamney 1980; Buitelaar 1993). So stammt die einzige genuin soziologische Studie zum Fasten von Joseph B. Tamney (1980), der im Jahr 1975 die Fastenpraxis von Muslim/innen auf der indonesischen Insel Java untersuchte. Durch eine standardisierte Befragung von 2783 Personen stellte er fest, dass das Fasten im Zuge fortschreitender Modernisierung zwar nicht an Bedeutung 
verliere, sich aber von einem Ausdruck der Frömmigkeit zu einem Ausdruck der Selbstkontrolle wandele.

Empirische Erkenntnisse über das zeitgenössische Fasten in Deutschland stammen bislang im Wesentlichen aus einer Umfrage der Forsa-Gesellschaft (Forsa 2019) im Auftrag der Krankenkasse DAK. Seit 2012 werden jährlich zu Beginn der vorösterlichen Bußzeit gut 1000 randomisiert ausgewählte Personen mit Wohnsitz in Deutschland befragt. Den entsprechenden Daten zufolge ist der Anteil derjenigen, die bereits mindestens einmal in ihrem Leben gefastet haben, zwischen 2012 und 2019 deutlich gestiegen: von gut der Hälfte auf knapp zwei Drittel der Bevölkerung. Unter den Fastenerfahrenen überwiegt der Anteil derjenigen, die bereits mehrfach gefastet haben, deutlich (45\%). Ihnen gegenüber steht ein Viertel der Befragten, die noch nie gefastet haben - und es auch nicht vorhaben. Zwischen den Fastenerfahrenen und den Menschen ohne Fastenabsicht findet sich eine vergleichsweise kleine Gruppe mit Fasteninteresse. Angesichts der offenbar hohen Popularität des Fastens in Deutschland ist zu vermuten, dass es nicht nur von spezifischen Personengruppen, sondern in allen Schichten, Alters- und Geschlechtsgruppen vergleichbar häufig praktiziert wird. Aufgrund der religiösen Tradition des Fastens kann jedoch davon ausgegangen werden, dass die Fastenerfahrung von der Religiosität und ihrer Konfessionszugehörigkeit einer Person abhängig ist. Die ersten Hypothesen der vorliegenden Studie lauten daher:

H1a: Die Fastenerfahrung einer Person ist unabhängig von ihren soziodemografischen Merkmalen.

H1b: Je höher die Religiosität einer Person, desto wahrscheinlicher ist eine Fastenerfahrung.

H1c: Konfessionsangehörige verfügen häufiger über eine Fastenerfahrung als Konfessionslose.

Wie oben erläutert, proklamieren verschiedene Religionen je spezifische Fastenzeiten, in denen sie ihren Gläubigen das Fasten mehr oder minder verbindlich nahelegen. Freilich steht es dem spätmodernen Individuum jedoch frei, auch außerhalb derartiger traditioneller Fastenzeiten zu fasten. Insbesondere das durch zahlreiche Publikationen populär gewordene Heil- oder Intervallfasten ist nicht an konfessionelle Fastenzeiten gebunden, sondern an der physiologischen Konstitution des fastenden Individuums orientiert. Angesichts weitreichender Säkularisierungs- und Freisetzungsprozesse in spätmodernen Gesellschaften ist sogar davon auszugehen, dass zeitgenössisches Fasten in nicht wenigen Fällen außerhalb traditioneller Fastenzeiten praktiziert wird. Gleichzeitig offenbart der einleitende Blick in die Presse, dass das Thema Fasten alljährlich zum Beginn der traditionellen Fastenzeiten - vor allem nach Karneval und im Ramadan - an Bedeutung für den medialen und öffentlichen Diskurs gewinnt. Es ist zu vermuten, dass die Entscheidung für oder gegen das Fasten während einer traditionellen Fastenzeit insbesondere von der Religiosität einer Person und von ihrer Konfessionszugehörigkeit abhängig ist. 
H2a: Je höher die Religiosität einer Person, desto wahrscheinlicher ist ihr Fasten während einer traditionellen Fastenzeit.

H2b: Konfessionsangehörige fasten häufiger während einer traditionellen Fastenzeit als Konfessionslose.

Für das zeitgenössische Fasten sind höchst unterschiedlichste Motivationen denkbar. Neben klassische religiöse Fastenmotivationen sind im Kontext des Heil- und Intervallfastens insbesondere gesundheitsorientierte Motivationen getreten. Es ist zu vermuten, dass eine religiöse Fastenmotivation in erster Linie von der Religiosität einer Person sowie von ihrer Konfessionszugehörigkeit abhängig ist.

H3a: Je höher die Religiosität einer Person, desto wahrscheinlicher ist eine religiöse Fastenmotivation.

H3b: Konfessionsangehörige äußern häufiger eine religiöse Fastenmotivation als Konfessionslose.

Wenn die Religiosität einer Person als Motivation für ihr Fasten begriffen werden kann, dann ist ebenfalls vorstellbar, dass das Fasten auf die Religiosität zurückwirkt. Von einer fastenbedingten Religiositätssteigerung kann dann gesprochen werden, wenn ein/e Fastende/r sich während des Fastens Gott näher fühlt, wenn er/sie häufiger betet als gewöhnlich oder wenn er/sie häufiger Gottesdienste besucht. Letzteres ist auch deshalb denkbar, weil insbesondere die christlichen Kirchen während der von ihnen proklamierten Fastenzeiten spezielle Liturgien anbieten, die eine höhere Popularität aufweisen könnten als alltägliche Gottesdienste. $\mathrm{Zu}$ vermuten ist ferner, dass ein gewisses Maß an Religiosität bereits vor dem Fasten gegeben sein muss, damit dieses einen Effekt auf die Religiosität haben kann.

H4a: Je höher die Religiosität einer Person, desto wahrscheinlicher ist eine fastenbedingte Religiositätssteigerung.

H4b: Konfessionsangehörige weisen häufiger eine fastenbedingte Religiositätssteigerung auf als Konfessionslose.

Worauf während des Fastens verzichtet wird, liegt in von Freisetzungsprozessen geprägten Gesellschaften ebenso in der selbstbestimmten Hand des Individuums wie die Entscheidung über den Zeitpunkt des Fastens. In Bezug auf die Verzichtsoptionen stechen in der oben zitierten Forsa-Umfrage insbesondere klassische Genussmittel wie Alkohol und Süßigkeiten heraus. Hinzu kommen mit Blockaden, Pessimismus, Lügen und ähnlichem die Verzichtsoptionen der evangelischen Fastenkampagne „Sieben Wochen ohne“. Unter dem Hashtag \#datenfasten finden sich in digitalen Medien darüber hinaus Initiativen, die zum weitgehenden Verzicht auf die private Nutzung von Internet, Computern und Smartphones während der Fastenzeit animieren. In vergleichbarer Manier riefen im Frühjahr 2019 die Partei Die Grünen und das Umweltbundesamt zum „Autofasten“ auf. ${ }^{3}$ Diese Beispiele verweisen auf eine Ausdifferenzierung von Fastenpraktiken: Neben religiöse Fastenmotivationen

\footnotetext{
3 Vgl. https://www.autofasten.de/.
} 
treten gesellschafts- und konsumkritische; neben den Verzicht auf Nahrungs- und Genussmittel tritt derjenige auf Medien und umweltschädigendes Verhalten. Es ist zu vermuten, dass diese neuen Fastenmotivationen und Verzichtsoptionen insbesondere bei einer nachwachsenden Generation von Fastenden zu finden sind. Als solche lassen sich sowohl junge Menschen verstehen als auch Fasteninteressierte, die zwar bislang noch nicht selbst gefastet haben, es aber zukünftig vorhaben.

H5a: Junge Menschen verzichten während des Fastens häufiger auf Medien und umweltschädigendes Verhalten als ältere. Auch Fasteninteressierte wollen dies häufiger tun, als Fastenerfahrene es bereits getan haben.

H5b: Junge Menschen und Fasteninteressierte äußern häufiger eine gesellschaftskritische Fastenmotivation als ältere Menschen und Fastenerfahrene.

\section{Daten und Operationalisierung}

\subsection{Datengrundlage}

Die im Folgenden präsentierten Daten wurden während der vorösterlichen Bußzeit des Jahres 2020 mittels einer offenen standardisierten Online-Befragung erhoben. Als Grundgesamtheit wurde zwar die erwachsene Bevölkerung der Bundesrepublik Deutschland angestrebt, jedoch konnte aus forschungspragmatischen Gründen keine an Repräsentativitätskriterien ausgerichtete Zufallsstichprobe erhoben werden. Um explorative Einblicke in zeitgenössische Fastenpraktiken zu gewinnen, wurde stattdessen ein selektives Sample akquiriert. Zur Teilnahme an der Befragung eingeladen wurden einerseits die Mitarbeitenden und Studierenden zweier deutscher Hochschulen: der FernUniversität in Hagen und der Katholischen Hochschule NRW. Beide zeichnen sich durch eine je spezifische und heterogene Studierendenschaft aus: Die Studierenden der FernUniversität in Hagen sind im Durchschnitt deutlich älter als Studierende an Präsenzuniversitäten und weisen ein breites Spektrum an Bildungsbiografien, beruflichen Erfahrungen und familiären Hintergründen auf. Da die Katholische Hochschule NRW ihre Studierenden auf die Ausübung kirchlicher Berufe vorbereitet, ist hier von einem besonders religionsaffinen Teil-Sample auszugehen. Um ein mögliches Bildungs-Bias zu begrenzen, wurde die Befragung andererseits über die Öffentlichkeitsarbeit der FernUniversität in Hagen beworben: Neben der Dissemination in sozialen Medien wurde auch über diverse Presseberichte und Interviews auf die Befragung aufmerksam gemacht. Auf diese Weise konnten 1908 Personen für eine Teilnahme gewonnen werden. Der Online-Fragebogen umfasste 36 Fragen. Das Gros der Fragen konnte in Form standardisierter Einoder Mehrfachauswahlen sowie als Likert-Skalen operationalisiert werden. Um das Spektrum der ausdifferenzierten Verzichtsoptionen möglichst vollständig zu erfassen, beinhaltete der Fragebogen darüber hinaus ein entsprechendes Freitextfeld. Die hier getätigten Antworten wurden mittels einer quantitativen Inhaltsanalyse kodiert. 


\subsection{Abhängige Variablen}

Als abhängige Variablen wurden fünf zentrale Aspekte des Fastens definiert. Erstens wurde erfasst, ob die Befragten über eigene Erfahrungen mit dem Fasten verfügen. Fall ja, wurde erhoben, ob sie erst einmal oder bereits mehrmals gefastet und wann sie dies zuletzt getan haben. Falls nein, wurde erhoben ob ein Fasteninteresse vorliegt. Dieses wird als starkes Fasteninteresse verstanden, wenn Befragte der Aussage zustimmen: „Ich möchte es unbedingt einmal ausprobieren“, sowie als unverbindliches Fasteninteresse, wenn sie der Aussage zustimmen: „Ich könnte es mir durchaus vorstellen“. Sowohl die Fastenerfahrenen als auch die Fasteninteressierten wurden zweitens nach den Motivationen für ihr (geplantes) Fasten gefragt. Hier konnten die Befragten ihre Zustimmung bzw. Ablehnung verschiedener potenzieller Fastenmotivationen angeben. Drittens wurden die Fastenerfahrenen danach gefragt, auf welche Nahrungsmittel, Genussmittel und Konsumgüter sie während ihres letzten Fastens verzichtet haben, und die Fasteninteressierten danach, worauf sie während ihres potenziellen Fastens verzichten wollen. Viertens wurde erhoben, ob die Befragten während einer traditionellen Fastenzeit gefastet haben bzw. dies vorhaben. Falls ja, wurde danach gefragt, um welche traditionelle Fastenzeit es sich dabei handelt. Fünftens wurden die Fastenerfahrenen nach den Effekten ihres letzten Fastens gefragt. Auch diese wurden durch eine Zustimmung bzw. Ablehnung verschiedener denkbarer Fasteneffekte erhoben.

\subsection{Unabhängige Variablen}

Als unabhängige Variablen wurden sowohl soziodemografische Merkmale als auch die Religiosität und Konfessionszugehörigkeit der Befragten erfasst (Tab. 1). Zu ersteren zählen Geschlecht, Alter, Wohnort, Bildungsniveau und Haushaltseinkommen. Mehr als zwei Drittel der Teilnehmer/innen sind weiblichen Geschlechts (68,6\%), ein knappes Drittel männlich (30,7\%). Der jüngste Teilnehmer ist zum Zeitpunkt der Befragung 16 Jahre alt, die älteste Teilnehmerin 87 (Mittelwert: 41,3; Median: 40; SD: 13,1). Da das ,säkulare Erbe der DDR“ (Pollack 2003) in den ostdeutschen Bundesländern zu einer nachhaltigen Einstellungsveränderung gegenüber religiösen Traditionen geführt hat, wurde der Wohnort der Teilnehmer/innen regional klassifiziert. In Ostdeutschland lebt gut jede/r zehnte Teilnehmer/in (11,0\%), in Westdeutschland leben mehr als vier Fünftel (80,8\%). Knapp die Hälfte der Teilnehmer/innen verfügt über ein monatliches Haushaltseinkommen von weniger als $3000 €(44,2 \%)$; einem knappen Drittel stehen zwischen 3000 und $5000 €$ zur Verfügung (31,8\%), dem restlichen Viertel mehr als $5000 €(24,0 \%)$. Das Bildungsniveau der Teilnehmer/innen wurde in Form eines Index berechnet, der sowohl den höchsten Schulals auch den höchsten berufsqualifizierenden Abschluss berücksichtigt (HoffmeyerZlotnik et al. 2010). Weniger als ein Prozent der Teilnehmer/innen weist dabei ein niedriges Bildungsniveau auf $(0,9 \%)$, zwei Fünftel ein mittleres $(38,4 \%)$ und drei Fünftel ein hohes Bildungsniveau (60,7\%). Das vergleichsweise hohe Bildungsniveau der Befragten mag auf die Akquisestrategie der Studie zurückzuführen sein. Mehr als die Hälfte der Teilnehmer/innen sind zum Zeitpunkt der Befragung Angehörige einer Konfession (57,5\%): jeweils gut ein Viertel der katholischen (28,3\%) 
Tab. 1 Unabhängige Variablen des untersuchten Samples

\begin{tabular}{|c|c|c|}
\hline Variable & $N$ & $\%$ \\
\hline \multicolumn{3}{|l|}{ Geschlecht } \\
\hline Männlich & 572 & 30,7 \\
\hline Weiblich & 1280 & 68,6 \\
\hline Divers & 14 & 0,8 \\
\hline \multicolumn{3}{|l|}{ Altersgruppe } \\
\hline Unter 30 Jahre & 406 & 21,3 \\
\hline 30-39 Jahre & 507 & 26,6 \\
\hline 40-49 Jahre & 408 & 21,4 \\
\hline 50-59 Jahre & 366 & 19,2 \\
\hline 60 Jahre und älter & 221 & 11,6 \\
\hline \multicolumn{3}{|l|}{ Wohnort } \\
\hline Ostdeutschland & 205 & 11,0 \\
\hline Westdeutschland & 1501 & 80,8 \\
\hline Außerhalb Deutschlands & 152 & 8,2 \\
\hline \multicolumn{3}{|l|}{ Bildungsniveau } \\
\hline Niedrig & 16 & 0,9 \\
\hline Mittel & 723 & 38,4 \\
\hline Hoch & 1126 & 60,7 \\
\hline \multicolumn{3}{|c|}{ Monatliches Haushaltseinkommen } \\
\hline Bis $1999 €$ & 397 & 23,3 \\
\hline $2000-2999 €$ & 358 & 21,0 \\
\hline $3000-4999 €$ & 542 & 31,8 \\
\hline Über $5000 €$ & 410 & 24,0 \\
\hline \multicolumn{3}{|l|}{ Konfession } \\
\hline Konfessionslos & 805 & 42,5 \\
\hline Katholisch & 536 & 28,3 \\
\hline Evangelisch & 482 & 25,4 \\
\hline Islamisch & 38 & 2,0 \\
\hline Sonstige & 35 & 1,8 \\
\hline \multicolumn{3}{|l|}{ Religiosität } \\
\hline Nicht-religiös & 427 & 22,5 \\
\hline Moderat nicht-religiös & 596 & 31,5 \\
\hline Moderat religiös & 466 & 24,6 \\
\hline Stark religiös & 406 & 21,4 \\
\hline
\end{tabular}

oder einer evangelischen Kirche (25,4\%). Die übrigen Konfessionsangehörigen verteilen sich auf den Islam $(2,0 \%)$ und sonstige Religionsgemeinschaften $(1,8 \%)$. Diese Werte entsprechen weitgehend der deutschen Gesamtbevölkerung. ${ }^{4}$ Die Religiosität der Befragten wurde multidimensional erfasst (Glock 1962; Pearce et al. 2013; Molteni und Biolcati 2018). In der kognitiven Dimension wurde der Glaube an Gott bzw. eine höhere Macht abgefragt, in der affektiven Dimension die religi-

\footnotetext{
4 Vgl. https://www.dbk.de/kirche-in-zahlen/kirchliche-statistik/ und https://www.ekd.de/ekd-statistik22114.htm.
} 
öse Selbsteinschätzung. In der praktischen Dimension wurden die Häufigkeit des Gottesdienstbesuchs und die Häufigkeit des Betens erfasst. Aus diesen vier Variablen wurde ein Religiositätsindex gebildet, der vier Ausprägungen annehmen kann (Bechert 2018): nicht-religiös (22,5\%), moderat nicht-religiös (31,5\%), moderat religiös $(24,6 \%)$ und stark religiös $(21,4 \%)$.

\section{Empirische Befunde}

Die Ergebnisse der vorliegenden Studie werden in drei Schritten dargestellt: Zunächst werden die Fastenpraktiken der Befragten deskriptiv beleuchtet. In einem zweiten Schritt werden die oben entwickelten Thesen regressionsanalytisch getestet. Fokussiert werden schließlich die Einflüsse von Konfessionszugehörigkeit und Religiosität auf zeitgenössische Fastenpraktiken in Deutschland.

\subsection{Fastenpraktiken}

\subsubsection{Fastenerfahrung}

Knapp drei Viertel der Befragten haben bereits mindestens einmal in ihrem Leben gefastet (72,4\%); knapp drei Fünftel mehrfach (58,7\%). Die letzte Fastenzeit dieser Fastenerfahrenen lag zwischen den Jahren 1960 und 2020 (Median: 2019, SD: 6,6). Ein Sechstel der Befragten hat zwar noch nie gefastet, zeigt aber ein entsprechendes Interesse $(16,7 \%)$. Von diesen Fasteninteressierten legt nur eine Minderheit ein starkes Fasteninteresse an den Tag $(24,0 \%)$, die Mehrheit $(76,0 \%)$ hingegen äußert ein eher unverbindliches Fasteninteresse (Abb. 1). Ein gutes Zehntel der Befragten schließlich weist weder Fastenerfahrung noch Fasteninteresse auf (10,9\%). Diese Werte decken sich im Wesentlichen mit der von Forsa (2019) untersuchten Zufallsstichprobe. An der vorliegenden Studie haben mithin nicht nur Personen teilgenommen, die sich in besonderer Weise für das Thema Fasten interessieren, sodass kein verzerrender Effekt durch ein Freiwilligen-Bias angenommen werden muss.

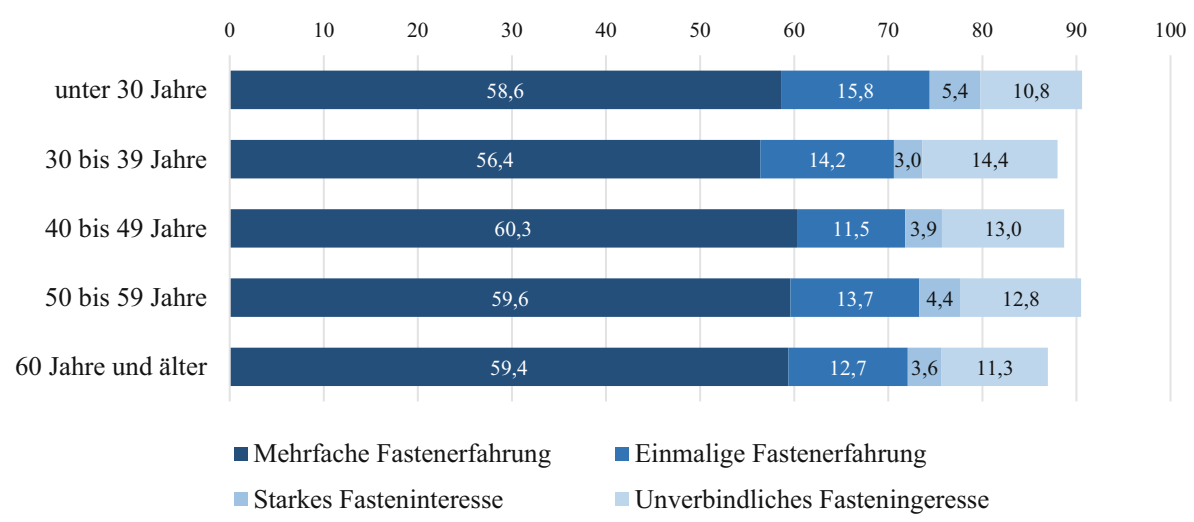

Abb. 1 Fastenerfahrung und -interesse nach Altersgruppen $(N=1908$; Angaben in Prozent $)$ 
Abb. 2 Fasten während traditioneller Fastenzeiten $(N=1696$; Angaben in Prozent)

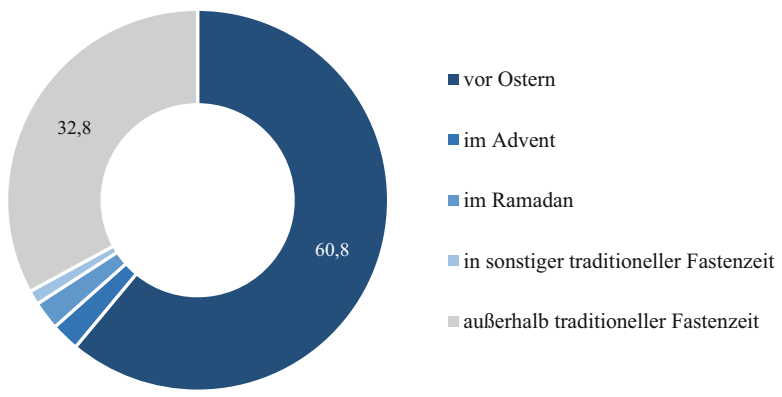

\subsubsection{Fastenzeiten}

Rund zwei Drittel der Befragten haben bzw. wollen während einer traditionellen Fastenzeit fasten (67,2\%). Auch in der späten Moderne sind es folglich Konfessionen, die verbindlich wirkende Anfangs- und Endpunkte des Fastens proklamieren. Diese institutionell verbrieften Fastenzeiten mit langer Tradition erfüllen für die Befragten eine kontingenzmindernde Funktion: Jeweils mehr als drei Fünftel stimmen den Aussagen zu, dass traditionelle Fastenzeiten es leichter machten, ,mit dem Verzicht zu beginnen“ (60,8\%) und diesen ,bis zum Ende der Fastenzeit durchzuhalten“ $(60,4 \%)$. Von Bedeutung im hier untersuchten Sample ist insbesondere die vorösterliche Bußzeit, in der 60,8\% der Fastenerfahrenen und Fasteninteressierten zuletzt gefastet haben bzw. zukünftig fasten wollen. Andere traditionelle Fastenzeiten spielen kaum eine Rolle (Abb. 2).

\subsubsection{Fastenmotivation}

Das Spektrum möglicher Fastenmotivationen lässt sich zu vier Gruppen zusammenfassen, von denen eine besonders heraussticht (Abb. 3): Annähernd neun von zehn Befragten geben eine subjektbezogene Motivation an $(88,9 \%)$. Hierzu zählen eine Verbesserung des körperlichen Wohlbefindens $(81,0 \%)$, eine Verbesserung des seelischen Wohlbefindens $(68,0 \%)$ und - mit deutlichem Abstand - der Wunsch, sich selbst zu bestrafen $(1,1 \%)$. Seltener findet sich eine gesellschaftskritische Fastenmotivation (34,6\%), zu der Konsumkritik (26,5\%) ebenso zählt wie Umwelt- und Naturschutz $(23,5 \%)$. Eine religiöse Fastenmotivation wird lediglich von rund jedem sechsten Befragten geäußert (17,0\%). Sie verstehen Fasten entweder als religiöse Pflicht (12,9\%) oder als Möglichkeit, den eigenen Glauben zu stärken (11,7\%). Die geringste Rolle spielt eine traditionsorientierte Fastenmotivation: Nur 8,3\% der Befragten stimmen der Aussage zu, gefastet zu haben bzw. fasten zu wollen, ,weil Menschen es schon immer tun“. Dieser Befund deckt sich mit Forschungsergebnissen zu anderen (ursprünglich) religiösen Praktiken in spätmodernen Gesellschaften: Beispielsweise geben auch Pilger/innen subjektbezogene Motivationen für ihre Pilgerschaft deutlich häufiger an als religiöse (Gamper und Reuter 2012; Gamper 2016). 
Ich habe gefastet bzw. ich möchte fasten...

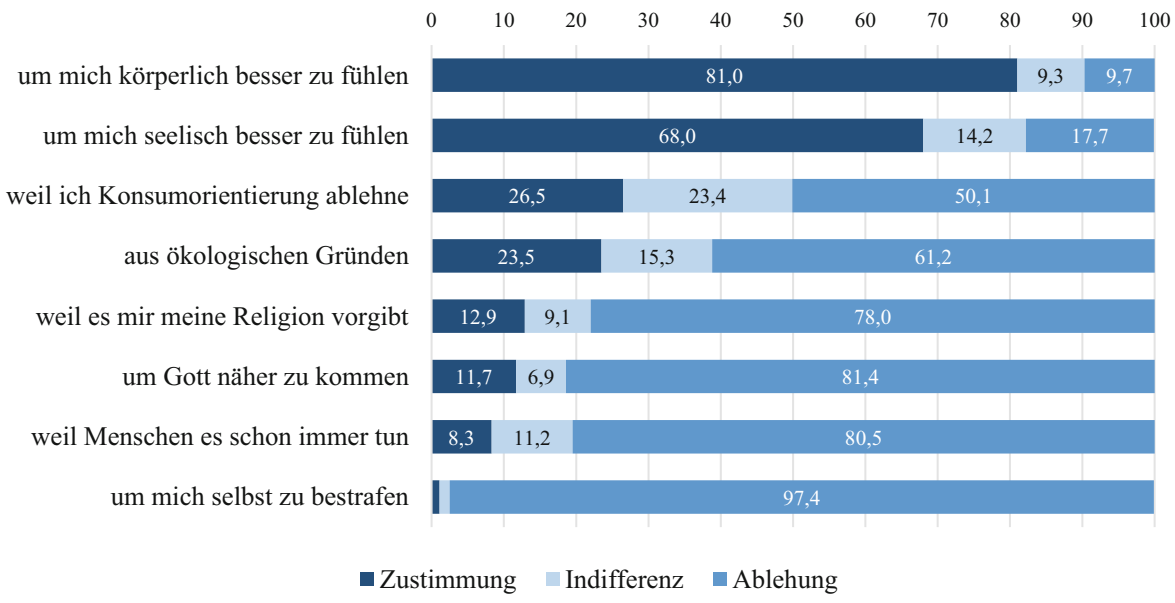

Abb. 3 Fastenmotivation ( $N=1699$; Angaben in Prozent, Mehrfachantworten möglich)

\subsubsection{Verzichtsoptionen}

Am häufigsten verzichten die Befragten während des Fastens auf Genussmittel (Abb. 4). Hierzu zählen in erster Linie Süßigkeiten (62,4\%) und Alkohol (54,5\%), mit einigem Abstand auch Rauchen (15,2\%), Drogen (11,4\%) und Kaffee (2,7\%). Insgesamt geben $86,1 \%$ der Befragten an, auf mindestens eines dieser Genussmittel verzichtet zu haben bzw. verzichten zu wollen. Auffällig sind diesbezüglich die Unterschiede zwischen Fastenerfahrenen und Fasteninteressierten: Auf Süßigkeiten will man offenbar häufiger verzichten, als man es tatsächlich tut; auf Alkohol hingegen haben die Fastenerfahrenen bereits häufiger verzichtet, als es die Fasteninteressierten vorhaben. Deutlich seltener wird auf Nahrungsmittel verzichtet (38,5\%). Hierzu zählen traditionell Fleisch $(32,9 \%)$, aber in jüngerer Zeit auch Kohlenhydrate $(5,8 \%)$ oder gar feste Nahrung im Allgemeinen (2,7\%). Insgesamt 13,8\% der Befragten verzichten außerdem auf den Konsum von Medien. Hierzu zählen das sogenannte „Datenfasten“, also der (weitgehende) Verzicht auf private Computer- und Internetznutzung (8,4\%), sowie der Verzicht auf Fernsehen (7,8\%). In Bezug auf derartige Medien sind die Unterschiede zwischen Fastenerfahrenen und Fasteninteressierten am augenfälligsten: Nur 10,4\% der Fastenerfahrenen geben an, tatsächlich auf Medien verzichtet zu haben, wohingegen 28,5\% der Fasteninteressierten angeben, dies zukünftig tun zu wollen. Einige Befragte verzichten während ihrer Fastenzeit schließlich auf umweltschädigendes Verhalten wie Autofahren $(3,3 \%)$ und den Verzehr tierischer Produkte $(2,9 \%)$.

Die quantitative Inhaltsanalyse eines Freitextfelds zum tatsächlichen bzw. beabsichtigten Verzicht offenbart darüber hinaus ein weites Spektrum individueller Verzichtsoptionen. Jeweils mehrfach, aber von weniger als einem Prozent der Befragten genannt wurden in Bezug auf die Ernährung der Verzicht auf Gluten, Fett, Salz, Fastfood, Softdrinks, verarbeitete Lebensmittel, reichhaltiges Essen, Frühstück 


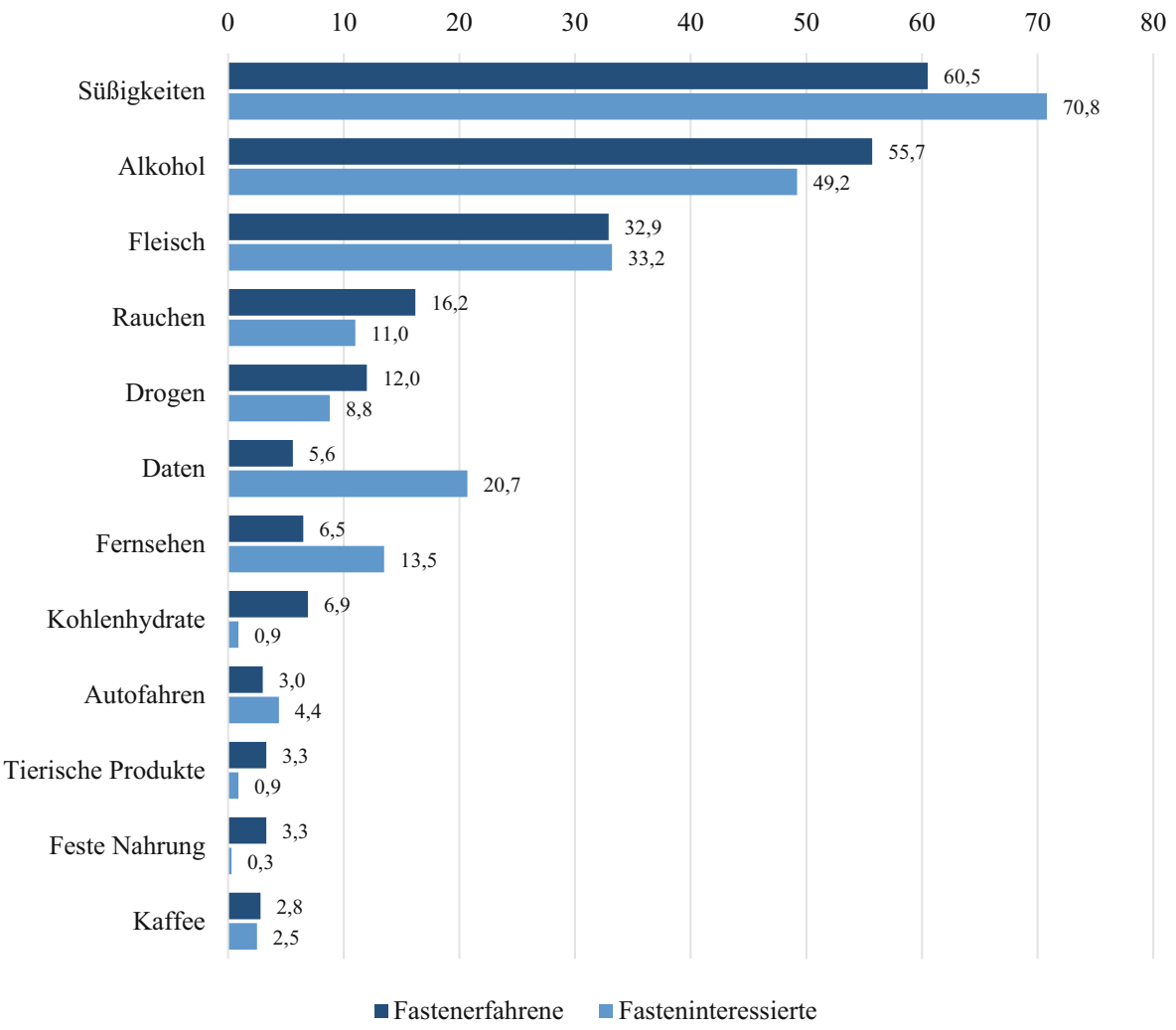

Abb. 4 Verzicht während des Fastens ( $N=1700$; Angaben in Prozent, Mehrfachantworten möglich)

Während des Fastens habe ich...

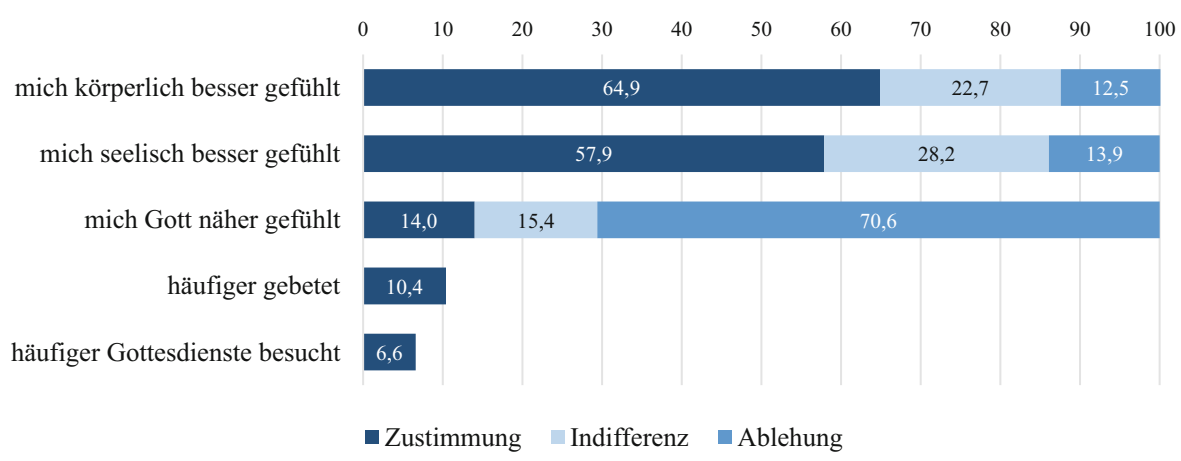

Abb. 5 Fasteneffekte ( $N=1381$; Angaben in Prozent, Mehrfachantworten möglich) 
und Abendbrot. In Bezug auf das Konsumverhalten wurde der Verzicht auf Shoppen, Veranstaltungsbesuche, Restaurantbesuche, Fliegen, Plastik, Produkte von Nestlé, Fernsehkrimis und Hörbücher genannt. In Bezug auf spezifische Praktiken wurden Grübeln, Hektik, Lästern, Meckern, Lügen, Streiten, Faulheit, Telefonieren, Benutzung von Aufzügen und Rolltreppen, Musik, Sex und Masturbation erwähnt. Ein Befragter möchte zudem auf seine Wohnung verzichten und während der Fastenzeit in einem Zelt leben.

\subsubsection{Fasteneffekte}

Die Fastenerfahrenen wurden schließlich zu den Effekten ihres letzten Fastens befragt (Abb. 5). Diese korrespondieren mit dem Wunsch nach einem verbesserten körperlichen und/oder seelischen Wohlbefinden, der - wie oben erläutert - die häufigste Fastenmotivation darstellt. So stimmen knapp zwei Drittel der Aussage zu, sie hätten sich ,während der Fastenzeit körperlich besser gefühlt“ (64,9\%); deutlich

Tab. 2 Konstanten des Fastens

\begin{tabular}{|c|c|c|c|}
\hline & $\begin{array}{l}\text { Verzicht auf } \\
\text { Genussmittel }\end{array}$ & $\begin{array}{l}\text { Subjektbezogene } \\
\text { Fastenmotivation }\end{array}$ & $\begin{array}{l}\text { Steigerung des körper- } \\
\text { lichen Wohlbefindens }\end{array}$ \\
\hline \multicolumn{4}{|l|}{ Geschlecht } \\
\hline Männlich $(N=572)$ & 88,0 & 87,2 & 60,4 \\
\hline Weiblich $(N=1280)$ & 85,4 & 89,6 & 66,1 \\
\hline \multicolumn{4}{|l|}{ Altersgruppen } \\
\hline Unter 30 Jahre $(N=406)$ & 81,0 & 88,3 & 60,3 \\
\hline 30-49 Jahre $(N=915)$ & 85,8 & 88,5 & 65,6 \\
\hline 50 Jahre und älter $(N=587)$ & 90,1 & 89,9 & 67,1 \\
\hline \multicolumn{4}{|l|}{ Wohnort } \\
\hline Westdeutschland $(N=1501)$ & 84,1 & 89,4 & 67,8 \\
\hline Ostdeutschland $(N=205)$ & 86,7 & 88,7 & 64,0 \\
\hline \multicolumn{4}{|l|}{ Bildungsniveau } \\
\hline Niedrig bis mittel $(N=729)$ & 86,2 & 89,4 & 66,7 \\
\hline $\operatorname{Hoch}(N=1126)$ & 85,9 & 88,7 & 63,3 \\
\hline \multicolumn{4}{|l|}{ Haushaltseinkommen } \\
\hline Bis $1999 €(N=397)$ & 83,7 & 87,6 & 62,0 \\
\hline $2000-2999 €(N=358)$ & 83,8 & 90,2 & 59,5 \\
\hline $3000-4999 €(N=542)$ & 87,2 & 90,5 & 70,7 \\
\hline $5000 €$ und mehr $(N=410)$ & 86,6 & 88,9 & 65,5 \\
\hline \multicolumn{4}{|l|}{ Religiosität } \\
\hline Nicht-religiös $(N=427)$ & 87,3 & 86,9 & 64,0 \\
\hline Moderat nicht-religiös $(N=596)$ & 86,3 & 91,1 & 66,4 \\
\hline Moderat religiös $(N=466)$ & 85,8 & 88,8 & 68,0 \\
\hline Stark religiös $(N=406)$ & 85,3 & 87,9 & 60,5 \\
\hline \multicolumn{4}{|l|}{ Konfessionszugehörigkeit } \\
\hline Konfessionsangehörige $(N=1091)$ & 89,0 & 92,2 & 68,0 \\
\hline Konfessionslose $(N=805)$ & 85,6 & 88,5 & 64,6 \\
\hline
\end{tabular}

Angaben in Prozent 
mehr als die Hälfte haben sich ,seelisch besser gefühlt“ (57,9\%). Darüber hinaus hat sich jede/r siebte Befragte während des Fastens „Gott näher gefühlt“ (14,0\%). Bei ihnen kann von einer Religiositätssteigerung in der affektiven Dimension von Religiosität gesprochen werden. Seltener findet sich eine Religiositätssteigerung in der praktischen Dimension: Nur jede/r Zehnte hat während des Fastens häufiger gebetet als normalerweise $(10,4 \%)$, jede/r Fünfzehnte hat häufiger Gottesdienste besucht (6,6\%). Das spätmoderne Fasten weist mithin eine nur geringe Anbindung an religiöse Kernpraktiken auf. Eine fastenbedingte Steigerung der Religiosität in mindestens einer der beiden Dimensionen zeigt sich bei 19,5\% der Fastenerfahrenen.

Zusammenfassend offenbart die deskriptive Analyse von Fastenpraktiken drei wesentliche Konstanten des zeitgenössischen Fastens in Deutschland: den temporären Verzicht auf Genussmittel, eine subjektbezogene Fastenmotivation und eine fastenbedingte Steigerung des körperlichen Wohlbefindens. Diese drei Konstanten werden von den Befragten nicht nur am häufigsten genannt, sondern finden sich vergleichbar häufig in allen untersuchten Gruppen (Tab. 2). Von soziodemografischen Merkmalen werden sie ebenso wenig beeinflusst wie von der Religiosität und der Konfessionszugehörigkeit. Die übrigen Verzichtsoptionen, Fastenmotivationen und -effekte hingegen werden sehr wohl von klar identifizierbaren Faktoren determiniert, wie im folgenden Abschnitt gezeigt werden wird.

\subsection{Determinanten des Fastens}

Um die oben entwickelten Hypothesen zu testen und die Forschungsfrage nach dem Zusammenhang von Fasten und Religion zu beantworten, stehen im Folgenden sechs Aspekte der dargestellten Fastenpraktiken im Fokus: (1) die Fastenerfahrung, (2) das Fasten während traditioneller Fastenzeiten, eine (3) religiöse bzw. (4) gesellschaftskritische Fastenmotivation, (5) der Verzicht auf Medien und/oder Umweltschädigung und (6) die fastenbedingte Religiositätssteigerung. Zur Erklärung dieser Aspekte werden als Bestimmungsfaktoren die Religiosität und die Konfessionszugehörigkeit der Befragten in den Blick genommen; soziodemografische Merkmale und der Wohnort fungieren als Kontrollvariablen. Um den Einfluss dieser unabhängigen Variablen auf die dichotom operationalisierten Fastenaspekte ermitteln zu können, wurden sechs logistische Regressionsmodelle geschätzt (Tab. 3).

Wie in Hypothese H1a vermutet, weisen die dargestellten Logit-Koeffizienten darauf hin, dass die Fastenerfahrung unter den Befragten weitgehend unabhängig von soziodemografischen Merkmalen und dem Wohnort verteilt ist. Lediglich das Geschlecht beeinflusst die Fastenerfahrung: So haben 75,2\% der Frauen bereits mindestens einmal gefastet, aber nur $67,1 \%$ der Männer. Zusammen mit der Beobachtung, dass an der vorliegenden Studie deutlich mehr Frauen teilgenommen haben als Männer, lässt dieser Befund vermuten, dass Frauen nicht nur ein größeres Interesse am Fasten zeigen, sondern es auch häufiger praktizieren als Männer. Auch von der Konfessionszugehörigkeit wird die Fastenerfahrung beeinflusst: So verfügen 73,4\% der Konfessionsangehörigen über Fastenerfahrung, aber nur 65,8\% der Konfessionslosen. Deutlich stärker fällt der Zusammenhang zwischen Fastenerfahrung und Religiosität aus: Von den stark religiösen Befragten haben 87,2\% bereits min- 


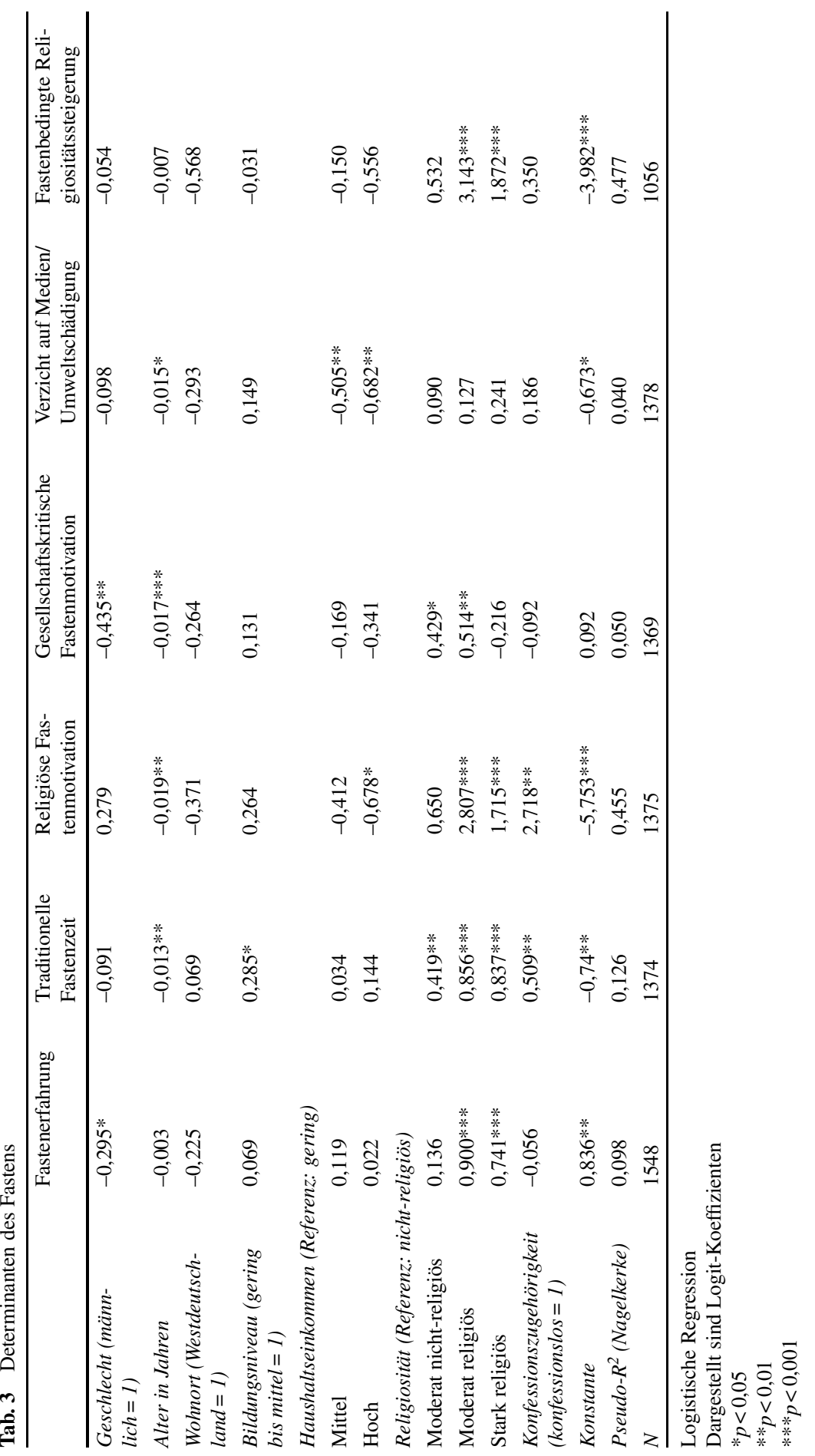


destens einmal im Leben gefastet, von den moderat religiösen 79,2\%, den moderat nicht-religiösen 64,4\% und den nicht-religiösen 61,8\%. Im Sinne der Hypothesen $\mathrm{H} 1 \mathrm{~b}$ und $\mathrm{H} 1 \mathrm{c}$ bringen eine Konfessionszugehörigkeit und eine mindestens moderate Religiosität also offenbar eine höhere Fastenaffinität mit sich. Bivariate Zusammenhanganalysen untermauern einen unabhängigen Zusammenhang von Religiosität und Konfessionszugehörigkeit mit der Fastenerfahrung, der nicht durch das Geschlecht determiniert wird.

Wie in den Hypothesen $\mathrm{H} 2 \mathrm{a}$ und $\mathrm{H} 2 \mathrm{~b}$ vermutet, wird auch der Zeitpunkt des Fastens von der Konfessionszugehörigkeit und der Religiosität einer Person determiniert. So fasten $70,1 \%$ der Konfessionsangehörigen während einer traditionellen Fastenzeit, aber nur 47,0\% der Konfessionslosen. Stark religiöse Befragte fasten zu $84,5 \%$ während einer traditionellen Fastenzeit, moderat religiöse zu 72,0\%, moderat nicht-religiöse zu 61,0\% und nicht-religiöse Befragte lediglich zu 50,6\%. Hinzu kommen zwei weitere Einflussfaktoren: Die Wahrscheinlichkeit, während einer traditionellen Fastenzeit zu fasten, nimmt mit zunehmendem Lebensalter leicht, aber mit hoher Signifikanz ab. Ein hohes Bildungsniveau bringt hingegen eine höhere Wahrscheinlichkeit mit sich, dass eine Person den Zeitpunkt des Fastens nicht selbstbestimmt wählt, sondern sich an einer traditionellen Fastenzeit orientiert. Auch hier bestätigen bivariate Zusammenhanganalysen den unabhängigen Einfluss von Religiosität und Konfessionszugehörigkeit.

Wie in den Hypothesen $\mathrm{H} 3 \mathrm{a}$ und $\mathrm{H} 3 \mathrm{~b}$ vermutet, ist das Vorliegen einer religiösen Fastenmotivation stark von der Konfessionszugehörigkeit und der Religiosität einer Person abhängig. Von den befragten Konfessionslosen geben gerade einmal $0,9 \%$ an zu fasten, um Gott näher zu kommen oder weil sie es als religiöse Pflicht empfinden; von den Konfessionsangehörigen hingegen äußern 19,4\% eine religiöse Fastenmotivation. Auch von der deutlichen Mehrzahl der Mitglieder einer Kirche oder Religionsgemeinschaft wird ihr Fasten mithin nicht explizit religiös begründet. Hier hat die individuelle Religiosität einen deutlich stärkeren Einfluss als die formale Mitgliedschaft. Auch nicht- oder moderat nicht-religiöse Personen nämlich geben nur in höchst seltenen Fällen eine religiöse Fastenmotivation an $(0,9$ bzw. $2,1 \%$ ); moderat religiöse Personen hingegen tun dies in $15,9 \%$ und stark religiöse in 52,6\% der Fälle. Eine religiöse Fastenmotivation setzt mithin ein hohes Religiositätsniveau voraus. Flankierend wird eine religiöse Fastenmotivation erneut vom Alter der Befragten determiniert: Hier ist bemerkenswert, dass die Wahrscheinlichkeit, aus religiösen Gründen zu fasten, mit dem Lebensalter leicht abnimmt. Hinzu kommt, dass ein höheres Haushaltseinkommen einen abträglichen Effekt auf die religiöse Fastenmotivation aufweist. Dies gilt tendenziell auch für eine fastenbedingte Religiositätssteigerung, also häufigeres Beten, häufigere Gottesdienstbesuche und/oder das Gefühl, Gott während des Fastens näher zu sein. Im Wesentlichen jedoch wird die fastenbedingte Religiositätssteigerung von der (Grund-)Religiosität determiniert: Sie zeigt sich bei gerade einmal $1,2 \%$ der Nicht-Religiösen und 1,4\% der moderat Nicht-Religiösen, jedoch bereits bei 16,6\% der Moderat-Religiösen und sogar bei $55,9 \%$ der stark religiösen Befragten. Im Sinne von Hypothese H4a steigt die Wahrscheinlichkeit einer fastenbedingten Religiositätssteigerung also mit höherer Religiosität - oder pointierter formuliert: Nur wer ohnehin schon religiös war, wird während des Fastens noch religiöser. Keinen Zusammenhang zeigt die Regressions- 
analyse hingegen mit der Konfessionszugehörigkeit einer Person. Im Gegensatz zu Hypothese H4b steigt die Wahrscheinlichkeit einer fastenbedingten Religiositätssteigerung also nicht durch eine Konfessionszugehörigkeit. Zu vermuten ist, dass dies durch starke interkonfessionelle Unterschiede zu erklären ist, auf die im folgenden Abschnitt eingegangen wird.

Schließlich sind die Hypothesen H5a und H5b zu testen, die auf neue Fastenmotivationen und Verzichtsoptionen abzielen. Im vorangegangenen Abschnitt wurde bereits deskriptiv gezeigt, dass Fasteninteressierte zukünftig häufiger aus gesellschaftskritischen Motiven fasten wollen, als Fastenerfahrene es in der Vergangenheit getan haben: Sie wollen häufiger aus ökologischen Gründen fasten und/oder weil sie die „Konsumorientierung unserer Gesellschaft“ ablehnen. Die Regressionsanalyse zeigt nun, dass sich derartige Fastenmotivationen häufiger bei Männern finden als bei Frauen und dass die Wahrscheinlichkeit für eine gesellschaftskritische Fastenmotivation mit zunehmendem Lebensalter sinkt. Ein ähnliches Bild zeigt sich beim Verzicht auf Medien und/oder umweltschädigendes Verhalten. So wollen neben den Fasteninteressierten vor allem jüngere Befragte auf Fernsehen und Internet, auf Autofahren und den Verzehr tierischer Produkte verzichten. Hinzu kommt, dass die Wahrscheinlichkeit für den Verzicht auf Medien und/oder Umweltschädigung mit einem höheren Haushaltseinkommen sinkt. Von der Religiosität und der Konfessionszugehörigkeit hingegen sind sowohl eine gesellschaftskritische Fastenmotivation als auch der Verzicht auf Medien und/oder Umweltschädigung weitgehend unabhängig. Dass neue Fastenmotivationen und Verzichtsoptionen sich somit in erster Linie bei Fasteninteressierten und jüngeren Menschen zeigen, spricht für eine nachwachsende Generation von Fastenden, die neue Gestaltungsaspekte in die jahrhundertealte Tradition des Fastens einbringt. Hierbei handelt es sich um Gestaltungsaspekte, die nicht religiös begründet werden, sondern sich auf säkulare Diskurse beziehen: auf Umweltschutz und Konsumkritik.

\subsection{Einflüsse von Religiosität und Konfessionszugehörigkeit}

Auch wenn die subjektive Begründung individuell gestalteter Fastenpraktiken von einer nachwachsenden Generation von Fastenden zunehmend mit säkularen Sinnkontexten verknüpft wird, zeigen sowohl die Regressionsanalyse als auch bivariate Zusammenhangsanalysen drei wesentliche Determinanten des zeitgenössischen Fastens in Deutschland: Neben dem Lebensalter werden die zentralen Aspekte des Fastens im Wesentlichen von der Religiosität und der Konfessionszugehörigkeit bestimmt. In Bezug auf die Religiosität lassen sich dabei klare Zusammenhänge erkennen: Je höher die Religiosität einer Person, desto wahrscheinlicher ist eine Fastenerfahrung, desto häufiger wird während traditioneller Fastenzeiten gefastet, desto wahrscheinlicher ist eine religiöse Fastenmotivation und desto häufiger ist die Religiosität während des Fastens gesteigert. Am deutlichsten fällt dieser Zusammenhang in Bezug auf die religiöse Fastenmotivation und die fastenbedingte Religiositätssteigerung aus. Beide setzen offenbar ein hohes Religiositätsniveau voraus (Abb. 6).

Der Einfluss der Konfessionszugehörigkeit auf das Fasten bedarf eines genaueren Blickes, da sich hier erhebliche interkonfessionelle Unterschiede zeigen: So haben 


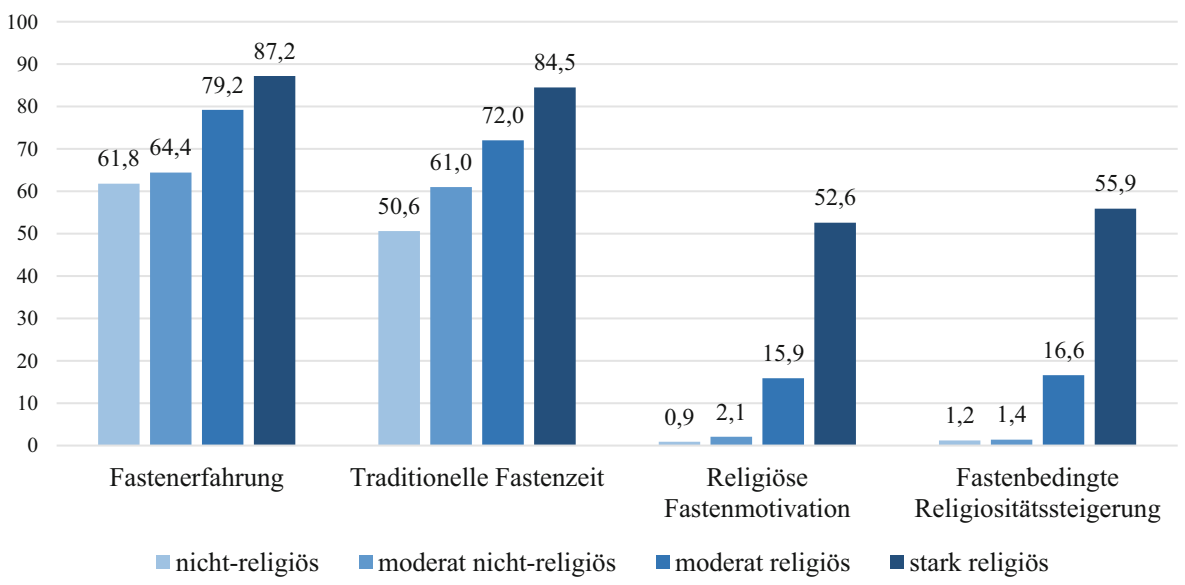

Abb. 6 Einflüsse der Religiosität auf das Fasten (Angaben in Prozent)

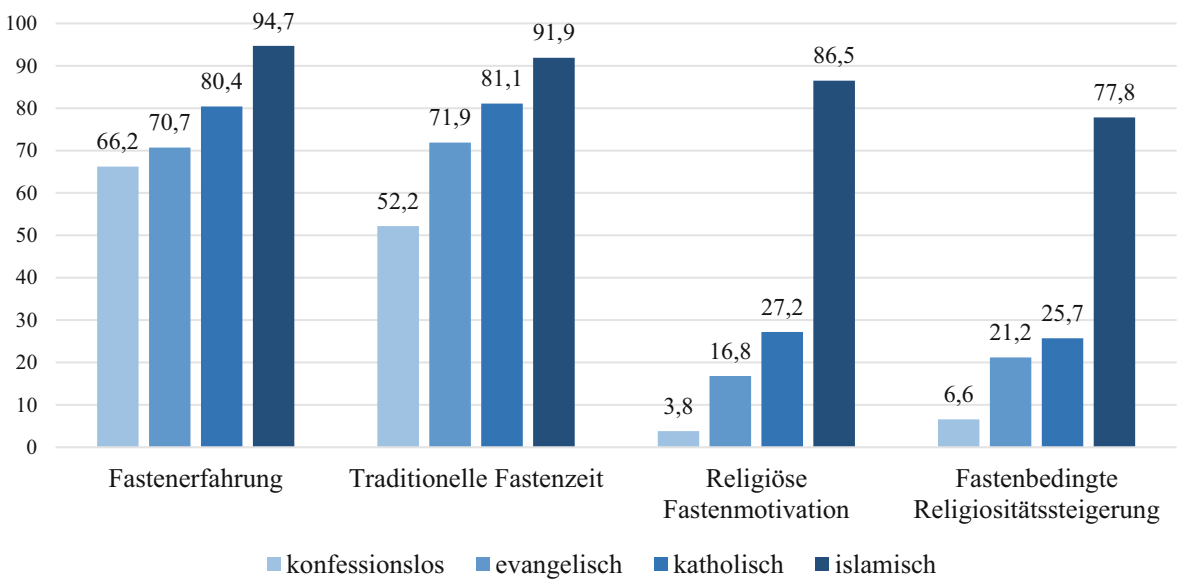

Abb. 7 Einflüsse der Konfession auf das Fasten (Angaben in Prozent)

$66,2 \%$ der Konfessionslosen bereits mindestens einmal in ihrem Leben gefastet, 70,7\% der Protestant/innen, 80,4\% der Katholik/innen und 94,7\% der Muslim/ innen. Dass dem muslimischen Fasten als einer der fünf Säulen des Islam ein besonderer Stellenwert zukommt, zeigt sich hier deutlich. Während einer traditionellen Fastenzeit fasten immerhin 52,2\% der Konfessionslosen, aber 71,9\% der Protestant/ innen, $81,1 \%$ der Katholik/innen und 91,9\% der Muslim/innen. Noch deutlicher zeigt sich der Unterschied zwischen christlichen Kirchen und Islam mit Blick auf die beiden folgenden Aspekte: Eine religiöse Fastenmotivation geben 3,8\% der Konfessionslosen an, 16,8\% der Protestant/innen und 27,2\% der Katholik/innen - aber $86,5 \%$ der Muslim/innen. Eine fastenbedingte Religiositätssteigerung lässt sich bei 6,6\% der Konfessionslosen, 21,2\% der Protestant/innen, 25,7\% der Katholik/innen und 77,8\% der Muslim/innen beobachten. In Bezug auf alle vier Aspekte zeigt 
sich mithin das gleiche Muster: von Konfessionslosigkeit über Protestantismus und Katholizismus hin zum Islam (Abb. 7).

\section{Diskussion}

Die empirischen Befunde der vorliegenden Studie zeigen einerseits, dass die wenigsten der hier Befragten expressis verbis eine religiöse Motivation für ihr Fasten äußern. Zwar vollziehen sie Fastenpraktiken, die mit denjenigen in vergangenen Jahrhunderten vergleichbar sind, deuten diese aber nur noch in seltenen Fällen als Religion. Dies könnte dafürsprechen, das zeitgenössische Fasten in Deutschland als säkulare Praktik zu begreifen, die vornehmlich in subjektzentrierten und gesundheitsorientierten Sinnkontexten vollzogen wird. Freilich jedoch verbietet es sich, subjektive Deutungen und individuelle Semantiken unmittelbar als Forschungsergebnisse zu übernehmen. Die Klassifikation einer beobachtbaren Praktik als religiös bzw. nicht-religiös muss vielmehr auf einer analytisch-interpretativen Ebene erfolgen. So wäre es beispielsweise zu kurz gegriffen, frühmodernes Fasten als religiöse Praktik zu begreifen, spätmodernes aber nicht. Gerade angesichts der für Modernisierungsprozesse typischen Verwebung von religiösem Handeln mit säkularen Kontexten dürfen auch solche Praktiken nicht aus dem religionssoziologischen Blick verloren werden, die von den sie vollziehenden Individuen nicht explizit als Religion benannt werden - ansonsten würden sie für soziologische Forschung ,unsichtbar“ (Luckmann 1991). Praktiken sollten daher weniger von ihrer Bezeichnung als eher von ihrem Wesen her analysiert werden. Dabei müssen institutionelle Rahmungen aus dem religiösen Feld ebenso berücksichtigt werden wie konfessionelle Traditionen und zugrundeliegende Mechanismen. In diesem Sinne zeigen drei Befunde der vorliegenden Studie denn auch andererseits einen deutlichen Zusammenhang von Fasten und Religion: erstens die persistente Verbindlichkeit der von Konfessionen proklamierten Fastenzeiten, die dazu führt, dass die Mehrzahl der Befragten in traditionellen Zeiträumen fastet; zweitens, dass mehr als zwei Drittel der Befragten fasten, um sich seelisch besser zu fühlen (wobei die Sorge um die Seele klassischerweise in den Zuständigkeitsbereich von Religion fällt), und drittens, dass Konfessionszugehörigkeit und Religiosität den weitreichendsten Einfluss auf zentrale Aspekte zeitgenössischer Fastenpraktiken ausüben; soziodemografische Kontrollvariablen spielen hier eine deutlich untergeordnete Rolle. Das Verhältnis von Fasten und Religion muss folglich differenziert betrachtet werden.

Um subjektive Deutungen, institutionelle Rahmungen und religiöse Traditionen integrativ analysieren zu können, bietet sich das Konzept der gelebten Religion (lived religion) an. Dieser Ansatz wird seit den 1990er-Jahren vornehmlich im angloamerikanischen Raum entwickelt (Hall 1997; Ammerman 2006; McGuire 2008; Neitz 2011), von der deutschsprachigen Religionssoziologie wurde er bislang jedoch nur in Ausnahmefällen rezipiert (bspw. Hennig 2017). Sein zentrales Anliegen besteht darin, durch eine möglichst offene und breite Forschungsperspektive die Gefahr zu minimieren, bestimmte Formen von Religion aufgrund theoretischer und methodischer Vorfestlegungen zu übersehen. In den Worten zweier Protagonistinnen des Lived-religion-Ansatzes: 
[W] e need to refocus our work in order to see the religion that often appears in unexpected places. [...] While belief and membership [...] are certainly a part of what lived religion entails, instead of starting from official organizations and formal membership, I want to begin with everyday practice; instead of taking the experts and official theology as definitive, I will join the lived religion scholars in arguing that we need a broader lens that includes but goes beyond those things. (Ammerman 2014, S. 189 f.)

We want to be open to the ways that people are sacralizing their daily lives, and to understand the various ways that people are using religious/spiritual practices in their daily lives to connect to traditions. (Neitz 2011, S. 54)

Aus Sicht der Lived religion verliert Religion auch in spätmodernen Gesellschaften durch Säkularisierungsprozesse nicht zwangsläufig an Bedeutung; vielmehr zeigt sie sich persistent in Gestalt vielfältiger gelebter Praktiken: „When one moves beyond adherence to institutions as the indicator, rather than seeing religion as disappearing under modernity, religion - as practices - appears to be proliferating“ (Neitz 2011, S. 47). Alle drei Zitate machen deutlich, dass es sich beim Terminus „Praktik“ um einen zentralen Begriff der gelebten Religion handelt. Er erlaubt es, Religion losgelöst von philosophischen oder theologischen Implikationen zu definieren und als Vollzugswirklichkeit zu verstehen, in der ein situativer Vollzug bestimmter Praktiken Religion immer wieder reproduziert (Hillebrandt 2012, S. 25, 51).

Häufig wird in der Religionssoziologie zwischen institutionen- und individualisierungstheoretischen Ansätzen unterschieden (Pollack 1995; Pickel 2011, S. 16ff.). In dieser Dichotomie möchte sich die gelebte Religion dezidiert nicht verorten. Aus ihrer Sicht nämlich bilden institutionalisierte Formen religiöser Organisation und individuell vollzogene religiöse Praktiken keinen Gegensatz. Vielmehr ist gelebte Religion auf vielfältige Weise mit institutionalisierten Formen von Religion verwoben: Erstere wird von letzterer gerahmt (Hall 1997, S. viii) und insofern gestützt, als religiöse Organisationen Begriffe, Gegenstände und Handlungsformate zur Verfügung stellen, auf die gelebte religiöse Praktiken in ihrem Vollzug zurückgreifen (Ammerman 2014, S. 157; Bender 2012, S. 280). Gelebte Religion tritt insofern in eine dezidierte Opposition zu institutionen- und organisationssoziologischen Forschungsansätzen, als sie - um es mit Schützeichel (2018) zu formulieren - deren ,,methodologischen Institutionalismus“ kritisiert, ,aus dessen Logik heraus sich religiöse Praxis als eine Applikation und nicht als eine eigenlogische Aneignung von Riten, Überzeugungen, Dogmen, Symbolen oder Glaubenslehren vollzieht“" (Schützeichel 2018, S. 94). Dieser antiinstitutionelle Impetus ist bei gelebter Religion allerdings wesentlich schwächer ausgeprägt, als es innerhalb der Religionssoziologie immer wieder der Fall ist, wenn sie sich auf Individualisierungsprozesse bezieht. Auch deshalb handelt es sich bei gelebter Religion keineswegs um eine rein individualistische Perspektive: „The forms of religion we need to be studying are not just located in individual consciousness. The way we understand the presence of religion in everyday life depends on recognizing it in the social processes where it is created and deployed" (Ammerman 2014, S. 194).

In der hier vorgestellten Perspektive stellt Religion kein distinktes Feld der Gesellschaft dar. Sie ist nicht als funktional differenzierte Sphäre zu verstehen, sondern 
als Praxisformation, die sich in allen Gesellschaftsbereichen beobachten lässt (Bender 2012, S. 281). Religion ist daher stets mit vielfältigen gesellschaftlichen und folglich auch mit säkularen Kontexten verwoben (Ammerman 2014, S. 195). Verortet wird gelebte Religion von allen hier zitierten Autor/innen im Alltäglichen. Dies ist einerseits nachvollziehbar, da es sich beim Alltag um denjenigen Ort handelt, ,an dem das Religiöse sein Eigengewicht gerade deshalb erhält, weil es mit den praktischen Dimensionen des Lebens verwoben ist. Religiosität ist nicht nur ein alltägliches Phänomen, sondern eingebettet in die Praxis des Alltags" (Schützeichel 2018, S. 94). Eine derartige Fokussierung auf die Alltagsrelevanz von Religion bringt jedoch andererseits ein gewichtiges Folgeproblem mit sich: Sie versperrt den Blick auf außeralltägliche Formen von Religion. Unter anderem aus der Pilgerforschung wissen wir aber, dass es auch und gerade außeralltägliche religiöse Praktiken sind, die unter den Bedingungen der späten Moderne eine hohe Popularität aufweisen (Heiser und Kurrat 2015; Kurrat und Heiser 2020). Auch für das Fasten ist seine Außeralltäglichkeit konstitutiv: Erst sein temporärer Charakter mit definierten Anfangs- und Endpunkten ermöglicht es dem/der Fastenden, von alltäglichen (Konsum-)Gewohnheiten zurückzutreten.

Aus der Lived-religion-Perspektive lässt sich Fasten als empirisches Beispiel dafür verstehen, dass religiöse Institutionen und die individuelle Gestaltung religiöser Praktiken keine dualistischen Gegensätze darstellen; erst ihr Zusammenspiel vermag die Popularität bestimmter religiöser Praktiken unter den Bedingungen der späten Moderne zu erklären. Freilich möchte der/die Fastende seine/ihre Fastenzeit heute selbstbestimmt gestalten. Wäre der Spielraum dazu nicht hinreichend groß, fiele die Popularität sicherlich geringer aus. Dies aber wäre auch dann der Fall, wenn keine Fastentradition vorläge, die von Konfessionen verbürgt würde und erst dadurch in der Lage ist, die Evidenz von Fastenpraktiken zu sichern. Individuell gestaltete religiöse Praktiken nämlich sind in verschärfter Form mit ihrer Kontingenz konfrontiert. Entscheidend ist daher, dass die institutionelle Rahmung dafür bürgt, dass es sich beim Fasten um eine ,richtige', jedenfalls in Hinblick auf erhoffte Wirkungen um eine effektvolle Praktik handelt. Daher lässt sich das zeitgenössische Fasten in Deutschland trotz der Bedeutungszunahme säkularer Fastenmotivationen und Verzichtsoptionen zusammenfassend als außeralltägliche Form gelebter Religion verstehen. Als religiöse Praktik, deren Popularität durch ein wechselseitig konstitutives Zusammenspiel von selbstbestimmter Gestaltung und individuellem Vollzug einerseits sowie von evidenzsichernder Tradition und institutioneller Rahmung andererseits erklärt werden kann.

\section{Fazit und Ausblick}

Auch in spätmodernen Gesellschaften wird das Fasten von vielen Menschen regelmäßig praktiziert; eine Beschränkung auf bestimmte Altersgruppen oder Schichten (gemessen an Bildung und Einkommen) lässt sich nicht erkennen. Im historischen Vergleich zeigt sich eine hohe Bedeutungsoffenheit des zeitgenössischen Fastens, in deren Rahmen wesentliche Aspekte vom fastenden Individuum selbstbestimmt gestaltet werden. Insbesondere Verzichtsoptionen und Fastenmotivationen differen- 
zieren sich dabei zusehends aus. Zwar verzichtet die deutliche Mehrheit der Befragten auf Genussmittel wie Süßigkeiten und Alkohol, doch reicht das Spektrum insgesamt von Fett bis Fastfood, von Frühstück bis Abendbrot, vom Shoppen bis zum Restaurantbesuch, von Plastik bis Fliegen, von Hektik bis Streiten, von der Aufzugbenutzung bis zum Geschlechtsverkehr - um nur einige Beispiele zu nennen. Die Analyse von Fastenmotivationen zeigt, dass Fasten mit stark divergierenden Sinnkontexten verknüpft wird: Es kann zweckrational als gesundheitsfördernd, wertrational als glaubensstärkend, affektuell als wohltuend für die Seele oder - in deutlich selteneren Fällen - traditional gedeutet werden. Trotz seiner individuellen Gestaltung und Deutung wird auch spätmodernes Fasten aber nicht jenseits eines tradierten institutionellen Rahmens praktiziert. Vielmehr proklamieren Konfessionen Fastenzeiten, die auch heute noch einen verbindlichen Charakter aufweisen: Nicht irgendwann fasten die Befragten, sondern im Wesentlichen vor Ostern oder im Ramadan.

Wie alle religiösen Praktiken ist auch das Fasten mit vielfältigen säkularen Kontexten verwoben. Hierfür lässt sich in den vorgestellten Daten eine Reihe von Beispielen finden: etwa der ökologisch motivierte Verzicht auf umweltschädigendes Verhalten, der gesellschaftskritische Verzicht auf Konsum und Medien oder der gesundheitsorientierte Verzicht auf Süßigkeiten und Tabak. Verzichtet wird während des Fastens häufig auf diejenigen Dinge, die in öffentlichen Diskursen ohnehin in der Kritik stehen. Auch deshalb unterliegen Fastenpraktiken einem permanenten Wandel. Dieser zeigt sich insbesondere an Fasteninteressierten, die bislang noch nicht selbst gefastet haben, es aber zukünftig vorhaben, und an jüngeren Fastenden. Beide Gruppen bringen neue Verzichtsoptionen und Deutungsmuster in die tradierte Praxis des Fastens ein. Diesen Wandel des Fastens zu begleiten, ist ein sicherlich lohnendes Unterfangen; für die Religionssoziologie, die anhand des Fastens mehr darüber erfahren kann, welche religiösen Praktiken auch unter den säkularen Bedingungen der späten Moderne hohe Popularitätswerte aufweisen. Eine solche Analyse populärer religiöser Praktiken kann dazu beitragen, den Wandel spätmoderner Religiosität insgesamt besser zu verstehen. Zentral scheint hier das spezifische Zusammenspiel von individueller Gestaltung und institutioneller Evidenzsicherung. Für den Ansatz der gelebten Religion erscheint die Fastenforschung lohnend, um seinen Fokus auf alltägliche Praktiken um außeralltägliche zu erweitern.

Im vorliegenden Aufsatz wurde der bislang umfangreichste Datensatz zum Fasten im dreisprachigen Raum analysiert. Die 1908 Befragten wurden vornehmlich aus hochschulischen Kontexten akquiriert, was einige Bias zur Folge hat, die möglicherweise die Generalisierbarkeit der hier diskutierten Daten und Schlussfolgerungen einschränken. Zunächst sind im untersuchten selektiven Sample Personen mit überdurchschnittlich hohem Bildungsniveau deutlich überrepräsentiert. Da sich der Anteil von Fastenerfahrenen und Fasteninteressierten jedoch weitgehend mit demjenigen deckt, der in einer repräsentativen Bevölkerungsumfrage ermittelt wurde (Forsa 2019), ist nicht davon auszugehen, dass Personen mit höherem Bildungsniveau häufiger fasten als solche mit einem niedrigen Bildungsniveau. Gleichwohl ist denkbar, dass sie ihr Fasten in Hinblick auf Verzichtsoptionen und/oder Fastenzeiten unterschiedlich gestalten. Auch Frauen sind im untersuchten Sample überrepräsentiert. Ob dies darin begründet ist, dass sie häufiger fasten als Männer, lässt sich auf 
Basis der hier untersuchten Daten nicht entscheiden. Darüber hinaus sind Westdeutsche überrepräsentiert, sodass sich die Forschungsfrage nicht adäquat beantworten lässt, ob das „,säkulare Erbe der DDR“ (Pollack 2003) zu einer nachhaltigen Einstellungsveränderung gegenüber der (ursprünglich) religiösen Praxis des Fastens geführt hat. Unterrepräsentiert sind schließlich nicht-christliche Konfessionen, sodass ein interreligiöser Vergleich von Fastenpraktiken nicht vorgenommen werden konnte. Zukünftige Fastenforschung sollte daher eine einschlägige repräsentative Zufallsstichprobe in den Blick nehmen. Angesichts der fortschreitenden Individualisierung von Fastenpraktiken scheint es darüber hinaus vielversprechend, die diskutierten Befunde durch qualitative Daten auszuleuchten. Die Verteilung von Konfessionslosigkeit, christlicher Konfessionszugehörigkeit und Religiosität im untersuchten Sample hingegen entspricht weitgehend derjenigen in der deutschen Gesamtbevölkerung. Daher kann davon ausgegangen werden, dass der herausgearbeitete Einfluss von Konfessionszugehörigkeit und Religiosität auf die Popularität und Gestaltung zeitgenössischer Fastenpraktiken sich auch jenseits der hier untersuchten Stichprobe zeigen wird.

Funding Open Access funding enabled and organized by Projekt DEAL.

Open Access Dieser Artikel wird unter der Creative Commons Namensnennung 4.0 International Lizenz veröffentlicht, welche die Nutzung, Vervielfältigung, Bearbeitung, Verbreitung und Wiedergabe in jeglichem Medium und Format erlaubt, sofern Sie den/die ursprünglichen Autor(en) und die Quelle ordnungsgemäß nennen, einen Link zur Creative Commons Lizenz beifügen und angeben, ob Änderungen vorgenommen wurden.

Die in diesem Artikel enthaltenen Bilder und sonstiges Drittmaterial unterliegen ebenfalls der genannten Creative Commons Lizenz, sofern sich aus der Abbildungslegende nichts anderes ergibt. Sofern das betreffende Material nicht unter der genannten Creative Commons Lizenz steht und die betreffende Handlung nicht nach gesetzlichen Vorschriften erlaubt ist, ist für die oben aufgeführten Weiterverwendungen des Materials die Einwilligung des jeweiligen Rechteinhabers einzuholen.

Weitere Details zur Lizenz entnehmen Sie bitte der Lizenzinformation auf http://creativecommons.org/ licenses/by/4.0/deed.de.

\section{Literatur}

Ahmad, Sartaj, Ashish Goel, Kham Maroof, Vaibhav Goel, und Mohamed Abid. 2012. Psycho-social behaviour and health benefits of Islamic fasting during the month of Ramadan. Journal of Community Medicine \& Health Education 2:178. https://doi.org/10.4172/2161-0711.1000178.

Ammerman, Nancy T. (Hrsg.). 2006. Everyday religion: observing modern religious lives. Oxford: University Press.

Ammerman, Nancy T. 2014. Finding religion in everyday life. Sociology of Religion 75:189-207.

Arbesmann, Rudolphus. 1929. Das Fasten bei den Griechen und Römern. Giessen: A. Töpelmann.

Bechert, Insa. 2018. Comparing religiosity cross-nationally. About invariance and the role of denomination. Zeitschrift für Religion, Gesellschaft und Politik 2:135-157.

Beck, Ulrich. 2008. Der eigene Gott. Friedensfähigkeit und Gewaltpotential der Religionen. Frankfurt a.M.: Verlag der Weltreligionen.

Bender, Courtney. 2012. Practicing religions. In The cambridge companion to religious studies, Hrsg. Robert A. Orsi, 273-295. Cambridge: University Press.

Buitelaar, Marjo. 1993. Fasting and feasting in Morocco. Women's participation in Ramadan. Oxford: Berg. 
Evangelische Kirche in Deutschland. 2020. 7 Wochen ohne. https://7wochenohne.evangelisch.de/. Zugegriffen: 24. Juni 2020.

Forsa. 2019. Fasten 2019. https://www.dak.de/dak/download/ergebnisbericht-2054020.pdf. Zugegriffen: 24. Juni 2020. Ergebnisbericht einer Umfrage im Auftrag der DAK Gesundheit.

Fritzsche, Bernardo. 2008. Religiöses Fasten. Gesundheit für Leib und Seele. Ostfildern: Patmos.

Gamper, Markus. 2016. Postpilger auf historischen Kulturwegen. Eine soziologische Studie zur Pilgerkultur auf dem Jakobsweg. In Kulturstraßen als Konzept. 20 Jahre Straße der Romanik, Hrsg. Andreas Ranft, Wolfgang Schenkluhn, und Nicole Thies, 121-139. Regensburg: Schnell + Steiner.

Gamper, Markus, und Julia Reuter. 2012. Pilgern als spirituelle Selbstfindung oder religiöse Pflicht? Empirische Befunde zur Pilgerpraxis auf dem Jakobsweg. In Doing Modernity - Doing Religion, Hrsg. Anna Daniel, Franka Schäfer, Frank Hillebrandt, und Hanns Wienold, 205-232. Wiesbaden: Springer VS.

Gebhardt, Winfried, Martin Engelbrecht, und Christoph Bochinger. 2005. Die Selbstermächtigung des religiösen Subjekts. Der ,spirituelle Wanderer“ als Idealtypus spätmoderner Religiosität. Zeitschrift für Religionswissenschaft 13:133-152.

Glock, Charles Y. 1962. On the study of religious commitment. Religious Education 57:98-110.

Grimm, Veronika E. 1996. From feasting to fasting. The evolution of a sin. London: Routledge.

Hall, David D. (Hrsg.). 1997. Lived religion in America. Toward a history of practice. Princeton: University Press.

Harris, Marvin. 1990. Wohlgeschmack und Widerwillen. Die Rätsel der Nahrungstabus, 3. Aufl., Stuttgart: Klett-Cotta.

Heiser, Patrick, und Christian Kurrat. 2015. Pilgern zwischen individueller Praxis und kirchlicher Tradition. Berliner Theologische Zeitschrift 32:133-158.

Hennig, Linda. 2017. Alltägliche Religiosität im Islam beobachten. In Religion soziologisch denken. Reflexionen auf aktuelle Entwicklungen in Theorie und Empirie, Hrsg. Kornelia Sammet, Heidemarie Winkel, 197-220. Wiesbaden: Springer VS.

Heun, E. 1972. Nahrungstabus und Fasten bei Naturvölkern. Ernährungsumschau 9:48-51.

Hillebrandt, Frank. 2012. Die Soziologie der Praxis und die Religion - Ein Theorievorschlag. In Doing Modernity - Doing Religion, Hrsg. Anna Daniel, Franka Schäfer, Frank Hillebrandt, und Hanns Wienold, 25-60. Wiesbaden: Springer VS.

Hocart, Arthur M. 1931. Fasting. In Encyclopedia of the Social Sciences, Bd. 5, Hrsg. Edwin R. Seligman, 144-146. New York: Macmillan.

Hoffmeyer-Zlotnik, Jürgen H.P., Axel Glemser, Christiane Heckel, Christian von der Heyde, und Helmut Quitt. 2010. Demographische Standards. Eine gemeinsame Empfehlung des ADM Arbeitskreis Deutscher Markt- und Sozialforschungsinstitute e.V., der Arbeitsgemeinschaft Sozialwissenschaftlicher Institute e. V. (ASI) und des Statistischen Bundesamtes, 5. Aufl., Wiesbaden: DeStatis.

Katholische Kirche. 2015. Die Fastenzeit: 40 Tage ohne. https://www.katholisch.de/glaube/unserkirchenjahr/40-tage-ohne. Zugegriffen: 24. Juni 2020.

Kurrat, Christian, und Patrick Heiser. 2020. Pilgrimage. In Wiley-Blackwell encyclopedia of sociology, Hrsg. George Ritzer. Hoboken: Wiley-Blackwell.

Laurioux, Bruno (Hrsg.). 1992. Tafelfreuden im Mittelalter. Kulturgeschichte des Essens und Trinkens in Bildern und Dokumenten. Stuttgart: Belser.

Luckmann, Thomas. 1991. Die unsichtbare Religion. Frankfurt a.M.: Suhrkamp.

McGuire, Meredith. 2008. Lived religion: Faith and practice in everyday life. Oxford: University Press.

Molteni, Francesco, und Ferruccio Biolcati. 2018. Shifts in religiosity across cohorts in europe: a multilevel and multidimensional analysis based on the European values study. Social Compass 65:413-432.

Musurillo, Herbert. 1956. The problem of ascetical fasting in the Greek patristic writers. Tradition 12:1-64.

Neitz, Mary J. 2011. Lived religion: Signposts of where we have been and where we can go from here. In Religion, spirituality and everyday practice, Hrsg. Giuseppe Giordan, William H. Swatos, 45-55. Dordrecht: Springer.

NRZ. 2019. Fasten: Jeder Zehnte plant Verzicht auf Alkohol und Süßes. https://www.nrz.de/panorama/ fasten-jeder-zehnte-plant-verzicht-auf-alkohol-und-suesses-id216596715.html. Zugegriffen: 24. Juni 2020. Neue Rheinzeitung.

Pearce, Lisa D., Jessica Halliday Hardie, und E. Michael Foster. 2013. A person-centered examination of adolescent religiosity using latent class analysis. Journal of the Scientific Study of Religion 52:57-79.

Pickel, Gert. 2011. Religionssoziologie. Eine Einführung in zentrale Themenbereiche. Wiesbaden: VS.

Pollack, Detlef. 1995. Was ist Religion? Probleme der Definition. Zeitschrift für Religionswissenschaft 3:163-190. 
Pollack, Detlef. 2003. Säkularisierung - ein moderner Mythos? Studien zum religiösen Wandel in Deutschland. Tübingen: Mohr.

Schützeichel, Rainer. 2018. Kontexte religiöser Praxis. In Religion im Kontext-Religion in Context: Handbuch für Wissenschaft und Studium, Hrsg. Annette Schnabel, Melanie Reddig, und Heidemarie Winkel, 85-102. Baden-Baden: Nomos.

Setzwein, Monika. 1997. Zur Soziologie des Essens. Tabu, Verbot, Meidung. Opladen: Leske + Budrich.

Suchy, Barbara. 1995. Die koschere Küche. Zur Geschichte der jüdischen Speisegesetze. In Speisen, Schlemmen, Fasten. Eine Kulturgeschichte des Essens, Hrsg. Uwe Schultz, 315-328. Leipzig: Insel.

Tamney, Joseph B. 1980. Fasting and modernization. Journal of the Scientific Study of Religion 2:129-137.

Teuteberg, Hans Jürgen. 1988. Magische, mythische und religiöse Elemente in der Nahrungskultur Mitteleuropas. In Wandel der Volkskultur in Europa, Bd. 1, Hrsg. Nils-Arvid Bringéus, 351-373. Münster: Coppenrath.

Wagner, Siegfried. 1986. Der Kampf des Fastens gegen die Fastnacht. Zur Geschichte der Mässigung. München: tuduv.

Wagtendonk, Kees. 1968. Fasting in the Koran. Leiden: Brill.

Zwingli, Ulrich. 1900. Von Freiheit der Speisen. Eine Reformationsschrift. Halle: Niemeyer. Hrsg. Otto Walther. 